\title{
A lower bound on the mass of Dark Matter particles
}

\author{
Alexey Boyarsky ${ }^{a, b}$, Oleg Ruchayskiy ${ }^{c}$, and Dmytro Iakubovskyi ${ }^{b}$ \\ ${ }^{a}$ ETHZ, Zürich, CH-8093, Switzerland \\ ${ }^{b}$ Bogolyubov Institute for Theoretical Physics, \\ Kiev 03680, Ukraine \\ ${ }^{c}$ École Polytechnique Fédérale de Lausanne, \\ Institute of Theoretical Physics, FSB/ITP/LPPC, \\ BSP 726, CH-1015, Lausanne, Switzerland
}

\begin{abstract}
We discuss the bounds on the mass of Dark Matter (DM) particles, coming from the analysis of DM phase-space distribution in dwarf spheroidal galaxies (dSphs). After reviewing the existing approaches, we choose two methods to derive such a bound. The first one depends on the information about the current phase space distribution of DM particles only, while the second one uses both the initial and final distributions. We discuss the recent data on dSphs as well as astronomical uncertainties in relevant parameters. As an application, we present lower bounds on the mass of DM particles, coming from various dSphs, using both methods. The model-independent bound holds for any type of fermionic DM. Stronger, model-dependent bounds are quoted for several DM models (thermal relics, non-resonantly and resonantly produced sterile neutrinos, etc.). The latter bounds rely on the assumption that baryonic feedback cannot significantly increase the maximum of a distribution function of DM particles. For the scenario in which all the DM is made of sterile neutrinos produced via non-resonant mixing with the active neutrinos (NRP) this gives $m_{\mathrm{NRP}}>1.7$ $\mathrm{keV}$. Combining these results in their most conservative form with the X-ray bounds of DM decay lines, we conclude that the NRP scenario remains allowed in a very narrow parameter window only. This conclusion is independent of the results of the Lyman-alpha analysis. The DM model in which sterile neutrinos are resonantly produced in the presence of lepton asymmetry remains viable. Within the minimal neutrino extension of the Standard Model (the $\nu$ MSM), both mass and the mixing angle of the DM sterile neutrino are bounded from above and below, which suggests the possibility for its experimental search.
\end{abstract}

\section{INTRODUCTION}

The nature of Dark Matter is one of the most intriguing questions of particle astrophysics. Its resolution would have a profound impact on the development of particle physics beyond the Standard Model.

Although the possibility of having massive compact halo objects (MACHOs) as a dominant form of DM is still under debate (see recent discussion in [1] and references therein), it is widely believed that Dark Matter is composed of non-baryonic particles. However, the Standard Model of elementary particles does not contain a viable Dark Matter particle candidate - a massive, neutral and long-lived particle. Active neutrinos, which are both neutral and stable, form structures in a top-down fashion [2, 3, 4, 5, 6], and thus cannot produce the observed quantity of early-type galaxies [see e.g. 7, 8]. Therefore, the DM particle hypothesis implies the extension of the Standard Model (SM).

The DM particle candidates may have very different masses (for reviews of DM candidates see e.g. [9, 10, 11, 12]): massive gravitons with the mass $\sim 10^{-19} \mathrm{eV}[13]$, axions with the mass $\sim 10^{-6} \mathrm{eV}$ [14], sterile neutrinos having mass in the keV range [15], sypersymmetric (SUSY) particles (gravitinos [16], neutralinos [17], axinos [18] with their masses ranging from $\mathrm{eV}$ to hundreds $\mathrm{GeV}$, supersymmetric Q-balls [19], WIMPZILLAs with the mass $\sim 10^{13} \mathrm{GeV}[20,21]$, and many others). Thus, the mass of DM particles becomes an important characteristic which may help to distinguish between various DM candidates and, more importantly, may help to differentiate among different models beyond the SM.

It was suggested in [22] that quite a robust and model-independent lower bound on the mass of DM particles can be obtained by considering phase space density evolution of compact astrophysical objects, most notably dwarf spheroidal satellites (dSphs) of the Milky Ways. The idea was developed further in a number of works (see e.g. 223, $24,25,26,27,28])$.

Another way to distinguish between various DM models, and particularly to put a bound on the DM mass, is the analysis of the Lyman- $\alpha$ (Ly- $\alpha)$ forest data [29, 30, 31]..$^{1}$ This method essentially constrains the possible shape of the power spectrum of density fluctuations at comoving scales $\sim$ Mpc. Assuming a DM model, (i.e. a particular primordial velocity distribution of DM particles), one can obtain a relationship between the DM particle mass in this model and

${ }^{1}$ Absorption feature by neutral hydrogen at $\lambda=1216 \AA$ at different redshifts in the spectra of distant quasars. 
the shape of the power spectrum, probed by Ly- $\alpha$.

Although very promising, the Ly- $\alpha$ method is very complicated and indirect. First of all, under the assumption that the distribution of the neutral hydrogen traces that of the DM, one can reconstruct the power spectrum of density fluctuations at redshifts $z \sim 2-5$ from the statistics of Lyman- $\alpha$ absorption lines. One can then perform a fit of the Lyman- $\alpha$ data (often together with the measurements of anisotropy of temperature of cosmic microwave background and the data of large-scale structure surveys), to extract the information about cosmological models. This is usually done by using the Monte-Carlo Markov chain technique [32]. At redshifts probed by Ly- $\alpha$, the evolution of structure has already entered the (mildly) non-linear stage. Therefore, to properly relate the measured power spectrum with the parameters of a given cosmological model one would have to perform a prohibitively large number of hydrodynamic numerical simulations. Therefore, various simplifying approximations have to be realized [30, 33, 34, 35, 36, 37, 38, 39].

Apart from these computational difficulties, the physics entering the Ly- $\alpha$ analysis is complicated, and not yet fully understood (see e.g. 40, 41, 42, 43, 44]). Moreover, the DM particles can significantly influence the background physics, further complicating the Ly- $\alpha$ analysis [45, 46, 47]. For a recent overview of the Ly- $\alpha$ method see e.g. [48].

The systematic uncertainties associated with both computational difficulties and complicated physics of Ly- $\alpha$ systems are not fully explored. Therefore, it is very important to have a lower mass bound on DM particles from more direct and simple considerations. In this paper we discuss the Tremaine-Gunn and related DM mass bounds based on the phase-space density considerations as well as possible ways to strengthen them for several DM models. The obtained phase-space density bounds are weaker yet comparable with Ly- $\alpha$ bounds and therefore provide an interesting alternative. We consider a class of the so-called "generic" DM models, where DM particles are produced thermally and decouple while being relativistic, thus having the (relativistic) Fermi-Dirac momentum spectrum. We also consider models of non-thermal DM production. In this case the primordial velocity spectrum of DM particles depends on the details of the production mechanism. We analyze the case when the velocity spectrum can be approximated by the rescaled Fermi-Dirac spectrum, or has two such components (a colder and a warmer one).

A very important example of such a DM particle is the sterile (right-handed) neutrino. Although known as a DM candidate for some 15 years [15], recently sterile neutrinos have attracted a lot of attention. It was shown [49] that if one adds three right-handed (sterile) neutrinos to the Standard Model, it is possible to explain simultaneously the data on neutrino oscillations (see e.g. 50, 51, 52] for a review) and the Dark Matter in the Universe, without introducing any new physics above electro-weak scale $M_{W} \sim 100 \mathrm{GeV}$. Moreover, if the masses of two of these particles are between $\sim 100 \mathrm{MeV}$ and electro-weak scale and are almost degenerate, it is also possible [53] to generate the correct baryon asymmetry of the Universe (see e.g. [54, [55]). The third (lightest) sterile neutrino can have mass in keV-MeV range ${ }^{2}$ and be coupled to the rest of the matter weakly enough to provide a viable (cold or warm) DM candidate.

This theory, explaining the three observed phenomena "beyond the SM" within one consistent framework, is called the $\nu M S M$ [49, [53] (see also 61]).

Although weakly coupled, the DM sterile neutrino in the $\nu \mathrm{MSM}$ can be produced in the correct quanities to account for all of the DM. There are several mechanisms of production: non-resonant active-sterile neutrino oscillations (nonresonant production mechanism, NRP) [15, 62, 63, 64, 65], resonant active-sterile neutrino oscillations in the presence of lepton asymmetry (resonant production mechanism, RP) [63, 66, 67, 68], decay of the gauge-singlet scalar field [69] (see also [57, 70, 71]). The Ly- $\alpha$ analysis of the sterile neutrino DM, produced via NRP scenario was performed in a number of works 72, 73, 74]. These bounds were recently revisited in [48], using the SDSS Ly- $\alpha$ dataset together with WMAP5 75]. The lower bound on the DM mass was found to be in this case $8 \mathrm{keV}$ (at 99.7\% CL). Ref. 48] also analyzed a more general case CWDM case : a mixture of NRP sterile neutrino with cold DM (see also [76]). These results were applied to the RP produced sterile neutrino in [77]. It was shown that the mass as low as $2 \mathrm{keV}$ is compatible with Ly- $\alpha$ data.

In this paper we will analyze in detail restrictions on the sterile neutrinos, produced via first two production mechanisms and briefly comment on the third one in the Discussion. In the case of non-resonant production the primordial velocity spectrum is approximately proportional to the Fermi-Dirac distribution [62, 78] (the exact spectrum was calculated in $[64,65])$.

The paper is organized as follows. In Section $\llbracket$ we review DM mass bounds, based on the phase-space density arguments. In Section [II we introduce the concept of maximal coarse-graining and propose a conservative modification of the original Tremaine-Gunn bound. In Section [V] we analyze new observational data on recently discovered dSphs (see [79, 80] and references therein), and use it to determine the phase-space density of these objects. Special attention is paid to determine various systematic uncertainties of measured values. Our results are summarized in Section $\nabla$ We conclude with the discussion of the results, analysis of possible uncertainties and outlook for the further improvement of the mass bounds in Section VI

\footnotetext{
${ }^{2}$ There are several interesting astrophysical applications of keV sterile neutrinos (see e.g. [45, 56, 57, 58, 59, 60] and references therein).
} 


\section{DM MASS LIMITS}

If the DM particles are fermions, there is a very robust bound on their mass. Namely, due to the Pauli exclusion principle, there exists the densest "packing" of the fermions in a given region of the phase space. Decreasing the mass of DM particles, one increases the number of them in a given gravitationally bound object, containing DM. The requirement that the phase-space density of the DM does not exceed that of the degenerate Fermi gas leads to the lower mass bound. For example, for a spherically symmetric DM-dominated object with the mass $M$ within the region $R$, one obtains the lower bound $m_{\mathrm{DEG}}$ on the DM mass by demanding that the maximal (Fermi) velocity of the degenerate fermionic gravitating gas of mass $M$ in the volume $\frac{4}{3} \pi R^{3}$ does not exceed the escape velocity $v_{\infty}=\left(\frac{2 G_{N} M}{R}\right)^{1 / 2}:$

$$
\hbar\left(\frac{9 \pi M}{2 g m_{\mathrm{DEG}}^{4} R^{3}}\right)^{1 / 3} \leq \sqrt{\frac{2 G_{N} M}{R}} \Rightarrow m_{\mathrm{DEG}}^{4} \geq \frac{9 \pi \hbar^{3}}{4 \sqrt{2} g M^{1 / 2} R^{3 / 2} G_{N}^{3 / 2}}
$$

Here and below $g$ denotes the number of internal degrees of freedom of DM particles, and $G_{N}$ is the Newton's constant. Such a consideration, applied to various DM dominated objects, leads to the mass bound, which we will call $m_{\text {DEG }}$ in what follows (see Table $\amalg$ below).$^{3}$

The above considerations assume that the dSphs are purely spherical systems. Analysis of [83] shows that ellipticity of stars in dSphs vary from $0.22_{-0.22}^{+0.18}$ for Leo IV to $0.80 \pm 0.04$ for Ursa Major I. Simulated DM halos on the other hand tend to have rather moderate ellipticity, $\epsilon_{D M} \lesssim 0.32[84] .{ }^{4}$ According to Appendix $\underline{\mathrm{A}}$, the ellipticity of DM halos can lower the resulting limit on $m_{\mathrm{DEG}}$ by $\lesssim 10 \%$.

The limit, obtained in such a way, is very robust, as it is independent of the details of the formation history of the system. The only uncertainties associated with it are those of astronomical nature: systematic errors in the determination of velocity and density distribution. All these issues will be discussed below (Section IV III).

For particular DM models (with the known primordial velocity dispersion) and under certain assumptions about the evolution of the system which led to the observed final state, this limit can be strengthened [22, 23, 24, 25, 85, 86, 87, 88. The argument is based on the Liouville's theorem (see e.g. [86, 89]) and assumes that the collapse of the system is disipationless and collisionless. The Liouville theorem states that the phase-space distribution function $f(t, x, v)$ does not change in the course of disipationless collisionless dynamics. The consequence of the Liouville theorem is that the function $f(t, x, v)$ "moves" in the phase-space, according to the Hamiltonian flow, and therefore its maximum (over the phase space) remains unchanged. Therefore, if one could determine the characteristics of a phase-space distribution function from astronomically observed quantities (in the first place average density $\bar{\rho}$ and velocity dispersion $\sigma)^{5}$ in dSphs (or any other DM dominated objects), the Liouville theorem would allow to connect the measured values with the primordial properties of DM particles.

One such characteristics of the phase-space distribution is its maximum. Any physical measurement can probe only the phase-space distribution, averaged over some phase-space region - a coarse-grained phase-space density (PSD) (as opposed to exact or fine-grained PSD). Such a coarse-grained PSD, averaged over phase-space cells $\Delta \Pi(x, v)$ centered around points $(x, v)$ in the phase space, is defined via

$$
\bar{f}(t, x, v)=\frac{1}{\operatorname{vol}(\Delta \Pi)} \int_{\Delta \Pi(x, v)} d \Pi^{\prime} f\left(t, x^{\prime}, v^{\prime}\right)
$$

(here $\operatorname{vol}(\Delta \Pi)$ is the volume of the phase-space cell). From the definition (2) it is clear that the maximal (over the whole phase space) value of the coarse-grained PSD $\bar{f}_{\text {max }}(t)$ cannot exceed the maximal value of the corresponding

\footnotetext{
3 The spatially homogeneous DM distribution is only an approximation. In reality one should consider self-gravitating degenerate fermionic gas. It is possible to show that, under some external conditions, the system of weakly interating fermions undergoes a first-orger phase transition to a nearly degenerate "fermion star" [81]. The existence of such objects may also have insteresting astrophysical applications [82].

4 Therefore it is hard to explain the ellipticity of stars in the most elongated dSphs, see the discussion in [83].

5 The quantity directly measured in astrophysical observations is the projection of stars' velocities $\vec{v}(R)$ along the line of sight. We will denote such a projection by $V(R)$ to distinguish it from the absolute value of the 3D velocity $v(r)$. The $1 \mathrm{D}$ velocity dispersion is defined as $\sigma(R)=\left\langle V^{2}(R)\right\rangle^{1 / 2}$ and is in principle the function of the projected radius $R$. However in the DM dominated objects, for $R$ greater than certain characteristic scale rotation curve flattens, $\sigma \approx$ const. It is this constant which is usually referred to as "velocity dispersion".
} 
fine-grained PSD. On the other hand, as a consequence of the Liouville theorem, the maximum of the fine-grained PSD $f_{\max }$ does not change in time. Thus, one arrives to the following inequality

$$
\bar{f}_{\max }(t) \leq f_{\max } .
$$

The inequality (3) allows to relate the properties of DM at present time $t$ with its primordial properties, encoded in $f_{\max }$. For example, if one assumes that initially DM particles possess relativistic Fermi-Dirac distribution function with some temperature $T_{\mathrm{FD}}$ (relativistically decoupled thermal relics):

$$
f_{\mathrm{FD}}(p)=\frac{g}{(2 \pi \hbar)^{3}} \frac{1}{e^{p / T_{\mathrm{FD}}}+1}
$$

and recovers from astronomical measurements that in the final state the coarse-grained PSD of the system is described by the isothermal sphere (see e.g. [89]) with a core radius $r_{c}$ and a $1 \mathrm{D}$ velocity dispersion $\sigma$, whose maximum is given by

$$
\bar{f}_{i s o, \max }=\frac{9 \sigma^{2}}{4 \pi G_{N}\left(2 \pi \sigma^{2}\right)^{3 / 2} r_{c}^{2}}
$$

the comparison of the maximum of the coarse-grained PSD (5) with its primordial (fine-grained) value leads to the so-called Tremaine-Gunn mass bound [22]:

$$
m_{\mathrm{FD}} \geq m_{\mathrm{TG}}, \quad \text { where } \quad m_{\mathrm{TG}}^{4} \equiv \frac{9(2 \pi \hbar)^{3}}{(2 \pi)^{5 / 2} g G_{N} \sigma r_{c}^{2}} .
$$

For the case of initial distribution (4) this bound is stronger than the one, based on the Pauli exclusion principle, by a factor $2^{1 / 4}[22]$. For different primordial DM distributions this difference can be significant (as we will demonstrate later). We would like to stress, though, that these stronger bounds make assumptions about the evolution of phasespace density, while the one, based on the Pauli exclusion principle does not assume anything about either primordial velocity distribution of the particles, or the formation history of the observed object and simply compares measured phase-space density with the maximally allowed for fermions.

Another characteristics of the phase-space distribution function is the "average phase-space density"

$$
Q \equiv \frac{\bar{\rho}}{\left\langle v^{2}\right\rangle^{3 / 2}},
$$

introduced in [26, 27]. The value of $Q_{f}$ (average PSD today) is simply defined in terms of the observed quantities $\bar{\rho}$ and $\left\langle v^{2}\right\rangle=3 \sigma^{2}$ and therefore serves as a convenient estimator of the PSD for any DM dominated object. One can calculate primordial $Q_{i}$ for an arbitrary homogeneous distribution function $f(p)$

$$
Q_{i}=\frac{g m^{4}}{(2 \pi \hbar)^{3}} \frac{\left(\int f(p) d^{3} p\right)^{5 / 2}}{\left(\int f(p) p^{2} d^{3} p\right)^{3 / 2}}
$$

and compare it with its value today $Q_{f}$. It was claimed in [26, 27] that $Q$ cannot increase during the evolution of DM:

$$
Q_{i} \geq Q_{f}
$$

Applying this inequality to the dSphs, one obtains several times stronger mass bound, than that of [22].

To illustrate the origin of the inequality (9), authors of [26, 27] noticed that in the case of the uniform monoatomic ideal gas, $Q$ is related to the usual thermodynamic entropy per particle (see Appendix B 1) and the inequality for $Q$ becomes a consequence of the second law of thermodynamics. Indeed, in this case one can see that

$$
\frac{S[f]}{N}=-\log \left(\frac{Q(\bar{\rho}, \sigma) \hbar^{3}}{m^{4}}\right)+\log C[f],
$$

where in the right hand side of (10) functional $C[f]$ does not depend on the average density and velocity of the DM particles. 
However, because of the long-range interaction of DM particles, the notion of Boltzmann entropy is well-defined only for the primordial DM distribution and not for the final state of DM evolution (see e.g. the discussion in [90]). Moreover, we will show below that in general the increase of entropy does not imply the decrease of $Q$. Indeed, the values of $C[f]$ are different for different types of phase-space distributions $f$ and therefore they can change with time if the shape of the (coarse-grained) distribution changes. Namely, even if initial $(i)$ and final $(f)$ states both satisfy relation (10) between the entropy and $Q\left(S_{i, f}=\log C_{i, f}-\log \frac{Q_{i, f} \hbar^{3}}{m_{\mathrm{DM}}^{4}}\right)$ from the second law of thermodynamics

$$
S_{f} \geq S_{i}
$$

it only follows that

$$
Q_{i} \geq Q_{f} \frac{C_{i}}{C_{f}}
$$

Therefore, in general, the inequality (9) does not follow from entropic considerations.

Moreover, the simple relation (10) between the entropy and $Q$ does not hold for the distributions we are interested in. For example, for the Fermi-Dirac distribution (4) one has:

$$
\frac{S}{N}=\text { const } \frac{m_{\mathrm{FD}}^{4}}{Q \hbar^{3}}
$$

(see Appendix B3 for details). The relation becomes even more complicated, if one considers DM candidates (e.g., sterile neutrinos, gravitinos), which are produced out of thermal equilibrium. In general, when the primordial distribution function depends on several parameters, both $Q$ and entropy are expressed through these parameters in a non-trivial way and the simple relation (10) does not hold. For example, this is the case when DM is produces in two stages and the DM distribution shape has two components: colder and warmer one. Physically interesting examples include: production of sterile neutrino in the presence of lepton asymmetry [66, 67, 68]; production of gravitino thermally at high temperatures (see e.g. [91, 92]) accompanied by non-thermal production via late decays of next-to-lightest supersymmetric particles (see e.g. [93]).

Keeping in mind the above considerations, one might be tempted to use the entropy of the system as an estimator of PSD and utilize the entropy increase (11) instead of the inequality on $Q$ to put a lower bound on the DM mass. However, unlike $Q$, which by definition is expressed solely in terms of measured quantities $\bar{\rho}$ and $\sigma$, the inequality (11) requires the knowledge of the phase-space distribution function in the final state (e.g. to determine the $C_{f}$ in the right-hand side of Eq. (10) or, more generally to express the entropy of the final state in terms of the observed quantities). This information cannot be simply deduced from astronomical observations. One possible way to formulate a conservative, robust inequality would be to find the maximal possible entropy for a given system with measured macroscopic parameters. However, it was shown in 86, 89, 94, 95] that such a maximum does not exist. Namely, for a gravitating system which usually consists of a compact core and a widely dispersed halo of finite mass, the total Boltzmann entropy of the system goes to infinity when the halo becomes infinite. Physically, the measured density and velocity dispersion characterize the inner part of the object. The astronomical observations do not usually probe the outskirts of gravitating systems (such as dSphs) and phase-space distributions (such as (5)) do not describe them properly. On the other hand, to compare with the homogenous initial system having a primordial velocity spectrum, we need to know an entropy of the whole system. The large (and unknown!) fraction of this entropy can be related to the outskirts. The entropy of the gravitating system depends on the precise state of the halo.

As a result, it is not possible to construct a simple and robust limit, using entropy considerations.

\section{MAXIMAL COARSE-GRAINING}

In view of the above arguments, to derive a conservative mass bound, in this work we will follow the original approach of Tremaine and Gunn [22] with some modification.

An important advantage of this approach is that the maximum of the phase space density is likely to be located in the inner, dense part of an object. Therefore, under this reasonable assumption, the results do not depend on the DM distribution in the outskirts (see the discussion above).

As discussed already, the coarse-grained phase-space distribution in the final state cannot be measured directly, and one has to make assumptions to deduce its maximum. A conservative way to minimize this uncertainty is to use the "maximally coarse-grained distribution". It is based on a simple fact that the mean value of a function, averaged over an arbitrary region cannot exceed its maximal value. Therefore, the average value of coarse-grained 
phase space density in a large phase-space volume can be taken as a conservative estimate of the $\bar{F}_{\text {max }}$, independent on assumptions about the actual form of phase-space distribution.

To this end we consider an (approximately spherically symmetric) gravitating system (having in mind a dwarf spheroidal galaxy), that has the mass $M(R)$ confined within the radius $R$. The phase-space volume, occupied by the DM particles, forming such a system can be approximated by

$$
\Pi_{\infty}=\left(\frac{4}{3} \pi\right)^{2} R^{3} v_{\infty}^{3},
$$

where we have introduced escape velocity $v_{\infty}^{2}$. The "coarsest" PSD is such that the averaging (2) goes over the whole phase-space volume: $\Delta \Pi=\Pi_{\infty}$ :

$$
\bar{F}=\frac{M}{\Pi_{\infty}}=\frac{9}{16 \pi^{2}} \frac{M}{R^{3} v_{\infty}^{3}}=\frac{3 \bar{\rho}}{4 \pi v_{\infty}^{3}}
$$

As an estimate for $R$ we take half-light radius $r_{h}$ (i.e. the radius where surface brightness profile falls to $1 / 2$ of its maximal value). Neglecting possible influence of ellipticity of stellar orbits (c.f. Appendix (A), assuming constant DM density within $r_{h}$ and isothermal distribution of stars [96], we obtain the following estimate on the average DM density within $r_{h}$ :

$$
\bar{\rho}=\frac{3 \log 2}{2 \pi} \frac{\sigma^{2}}{G_{N} r_{h}^{2}},
$$

Assuming isotropic velocity distributions, ${ }^{6}$ the escape velocity $v_{\infty}$ of the DM particles is related to the velocity dispersion $\sigma$ via $v_{\infty} \simeq \sqrt{6} \sigma$. In such a way we obtain the averaged PSD $\bar{F}$ :

$$
\bar{F}=\frac{M}{\Pi_{\infty}}=\frac{\bar{\rho}}{8 \pi \sqrt{6} \sigma^{3}} \approx \frac{3 \log 2}{16 \sqrt{6} \pi^{2} G_{N} \sigma r_{h}^{2}} \approx 1.25 \frac{M_{\odot}}{\mathrm{pc}^{3}}\left(\frac{\mathrm{km}}{\mathrm{sec}}\right)^{-3}\left(\frac{\mathrm{km} / \mathrm{sec}}{\sigma}\right)\left(\frac{1 \mathrm{pc}}{r_{h}}\right)^{2},
$$

which coincides with its maximal value (being flat).

As a consequence of Eq. (3), this "coarse-grained" PSD $\bar{F}$ is smaller than the $f_{\max }$ - the maximum value of fine-grained PSD, equal to its primordial value:

$$
\bar{F} \leq f_{\max } .
$$

Eq. (18) relates the observed properties of the DM-dominated systems (1.h.s.) with the microscopic quantity on the r.h.s. of inequality, which depends on the production mechanism of the DM.

In this paper we are mostly interested in two types of primordial momentum distribution. One is the relativistic Fermi-Dirac (4) with its $f_{\max }$ being equal to

$$
f_{\max , \mathrm{FD}}=\frac{g m_{\mathrm{FD}}^{4}}{2(2 \pi \hbar)^{3}}
$$

(we fix the overall normalization of the phase-space distribution function by the relation $M=\int d^{3} x d^{3} v f(t, x, v)$, where $M$ is the total mass of the system). Another one is an (approximate) form of the momentum distribution for sterile neutrinos, produced via non-resonant oscillations with the active ones [15, 62]. For the latter case we consider the velocity dispersion to $b^{7}$

$$
f_{\mathrm{NRP}}(p)=\frac{g \chi}{e^{p / T_{\nu}}+1} .
$$

\footnotetext{
6 This assumption seems to be correct for the DM particles, since numerical simulations of DM structures of different scales show that the velocity anisotropy $\beta(r) \equiv 1-\frac{\sigma_{\theta}^{2}+\sigma_{\phi}^{2}}{2 \sigma_{r}^{2}}$ tends to be zero towards the central region [97, 98, 99, 100, 101]. It is not clear whether $\beta$ equals to zero for stars in dSphs. The assumption of isotropy of stellar velocities leads to the cored density profiles 102, 103], therefore our estimate for $\bar{\rho}$ tends to be robust. This is confirmed by comparison of the estimate (16) with those, based on [104, 105, 106], where DM density profiles were obtained under the assumptions of different anisotropic distributions of stars in dSphs.

7 In reality the momentum distribution in the case of non-resonant production does not have thermal shape. The exact shape, taking into account contributions from primeval plasma at temperatures around QCD transition, can be computed only numerically 64 , 65]. The difference between the exact distribution and (20) does not exceed 20\%, which does not affect the mass bounds.
} 
The normalization constant $\chi$ is proportional to the mixing strength between active and sterile neutrinos and $T_{\nu}$ is the temperature of neutrino background $T_{\nu}(z)=(1+z) T_{\nu_{0}}$, related to the temperature of the CMB background today via $T_{\nu_{0}}=(4 / 11)^{1 / 3} T_{\mathrm{CMB}, 0}$. For the maximal value of distribution (20) we find

$$
f_{\max , \mathrm{NRP}}=\frac{g \chi m_{\mathrm{NRP}}^{4}}{2(2 \pi \hbar)^{3}} .
$$

From the definition (20) one can relate the normalization factor $g \chi$ to the DM abundance (see e.g. 78])

$$
\omega_{\mathrm{DM}} \equiv \Omega_{\mathrm{DM}} h^{2}=g \chi \frac{m_{\mathrm{NRP}}[\mathrm{eV}]}{94 \mathrm{eV}} .
$$

Therefore we can rewrite maximal value of the primordial phase-space density (22) as

$$
f_{\max , \mathrm{NRP}}=\frac{94 \omega_{\mathrm{DM}}}{2(2 \pi \hbar)^{3}} \frac{m_{\mathrm{NRP}}^{3}}{\mathrm{eV}^{3}}
$$

Notice, that unlike the Fermi-Dirac case, for the NRP scenario $f_{\text {max }}$ behaves as the third power of particle's mass.

In the presence of lepton asymmetry in primeval plasma the resonant production of sterile neutrinos becomes possible 66]. A possible lepton asymmetry, generated in the framework of the $\nu$ MSM and spectra of sterile neutrino DM were recently computed in [67, 68]. Qualitatively, these spectra contain a "cold" (resonant) component and a "warm" one, produced through non-resonant oscillations, analogously to the NRP scenario of [15]. The spectra as a whole become colder than in the NRP case (see e.g. Fig. 6 in [68]). The maxima of primordial phase-space distributions for these spectra are higher (sometimes significantly) than for spectra, produced in the NRP scenario (c.f. Fig. 5 in [68]). Therefore, in general mass bound for such a DM is expected to be weaker than that of the NRP scenario. The exact form of these spectra can be computed only numerically. We used a number of spectra ${ }^{8}$ to check those which satisfy the bound (18) or TG bound (see Section V).

Let us compare expression (17) with the original Tremaine-Gunn bound (maximum of the right hand side of Eq. (6)):

$$
F_{\mathrm{TG}}=\frac{9}{8 \pi^{2} \sqrt{2 \pi} G_{N} \sigma r_{c}^{2}}
$$

The values of $\bar{F}$ is smaller than $F_{\mathrm{TG}}$ by

$$
\frac{\bar{F}}{F_{\mathrm{TG}}}=\frac{\log 2 \sqrt{\pi}}{6 \sqrt{3}}\left(\frac{r_{c}}{r_{h}}\right)^{2} \approx 0.118\left(\frac{r_{c}}{r_{h}}\right)^{2}
$$

where $r_{c}$ and $r_{h}$ are the core radius of isothermal profile and the half-light radius, correspondingly. When comparing $\bar{F}$ and $F_{\mathrm{TG}}$ below, we take $r_{h} \simeq r_{c}$. Essentially, the difference between $\bar{F}$ and $F_{\mathrm{TG}}$ is due to the different assumed velocity distributions. While the Maxwell distribution was assumed in [22] (c.f. Eq. (5)), we assume constant velocity profile from escape velocity $v_{\infty}$ down to $v=0$ (as shown on the Fig. 1). The numerical factor in (25) is the ratio of areas under two velocity curves of Fig. 1. Translated into the mass bound, relation (25) means that for DM particles with distribution (4) one would obtain roughly $40 \%$ stronger mass bound by using the original Tremaine-Gunn bound, rather than $\bar{F}$ (and $\approx 60 \%$ stronger mass bound for the case of the distribution (201)).

Let us compare our new bound with the one, based of [26, 27]. Following the definition (77), we express the measured value $Q_{f}$ for a $\mathrm{dSph}$ through the observed quantities

$$
Q=\frac{\bar{\rho}}{\eta^{3}\left(3 \sigma^{2}\right)^{3 / 2}} \approx 14.83 \frac{M_{\odot}}{\mathrm{pc}^{3}}\left(\frac{\mathrm{km}}{\mathrm{sec}}\right)^{-3}\left(\frac{\mathrm{km} \mathrm{sec}^{-1}}{\sigma}\right)\left(\frac{1 \mathrm{pc}}{r_{h}}\right)^{2} \frac{1}{\eta^{3}},
$$

where $\eta$ is the scaling factor which accounts for the fact that the dark matter particles do not necessarily have the same velocity dispersion as the stars, $r_{h}$ is the half-light radius, $\sigma$ is the measured one-dimensional velocity dispersion of the stars and $\bar{\rho}$ is defined in (16). It was estimated in [26] that $\eta \approx 1$. In Eq. (26) we used the same value of $\bar{\rho}$ as in Eq. (17). For for the same dSph, $Q_{f}$ is bigger than $\bar{F}$ (given by expression (17)) by a factor $8 \pi \sqrt{2} / 3 \approx 11.85 \ldots$

\footnotetext{
${ }^{8}$ We are grateful to M. Laine and M. Shaposhnikov for providing these spectra to us.
} 


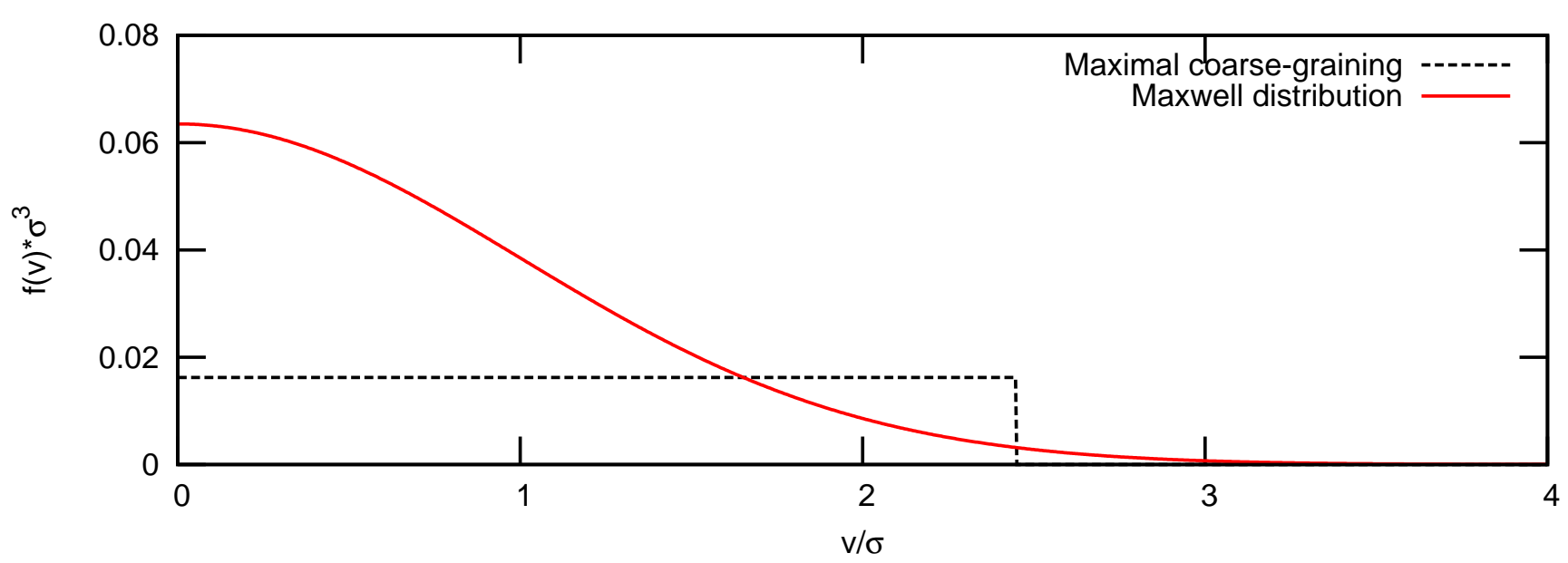

FIG. 1: Comparison of velocity profiles assumed in [22] (red solid line) and in this work (black dashed line).

On the other hand, for any initial momentum distribution $f(p)$ we should compare $Q_{i}$, given by Eq. (8), with the $f_{\max }^{(i)}$. For both types of distribution (4) and (20) the ratio of initial $Q_{i} / f_{\max }^{i}$ is given by

$$
\frac{Q_{i}}{f_{\max }^{(i)}}=\frac{4 \pi \zeta^{5 / 2}(3)}{5 \sqrt{15} \zeta^{3 / 2}(5)} \approx 0.973 \ldots
$$

As a result, a bound, based on the decrease of the average PSD $Q$ is stronger than $\bar{F}$ bound from the same object by a factor:

$$
\frac{f_{\max }^{(i)}}{Q_{i}} \frac{Q_{f}}{\bar{F}} \approx 12.176 \ldots
$$

(where again we put $\eta=1$ ). This leads to $\approx 1.87$ times stronger bound on the $m_{\mathrm{FD}}$ and $\approx 2.3$ times stronger bound for $m_{\mathrm{NRP}}$.

\section{ANALYSIS OF MEASURED VALUES}

Recently, a number of very faint, very dense dSphs were detected $[79,80,107,108,109,110,111]$. To calculate the mass limits, we used the data from two recent papers: [79, 80]. First of all, we should notice that although both of these papers provide the estimate of $Q$ for each object, they use different prescriptions for computing this value.

In [80] the quantity $Q$ is estimated inside the half-light radius $r_{h}$, using one-dimensional velocity dispersion $\sigma$ of stars:

$$
Q_{G i l}=\frac{\bar{\rho}}{\sigma^{3}}=\frac{3}{8 \pi G_{N} r_{h}^{2} \sigma} .
$$

Compared to our definition (17) $\bar{F}=\frac{\log 2}{2 \sqrt{6} \pi} Q_{\text {Gil }} \approx 0.045 Q_{\text {Gil }}$. Following [112] the authors of [79] define central density

$$
\rho_{0}=166 \sigma^{2} \eta^{2} / r_{c}^{2}
$$

where $\eta \sim 1$ is a numerical parameter, characterizing plausible density profiles (for details see [79, 112]). They used $\rho_{0}$ to define the quantity:

$$
Q_{S G} \equiv \frac{\rho_{0}}{\sigma^{3}}
$$

As a result for the same object $Q_{S G}$ is by a factor of 14.60 greater than $Q_{\text {Gil }}$.

Using the available information about dSph galaxies (refs. $[79,[80]$ and refs. therein), we calculate $\bar{F}$, trying also to estimate the errors. Several factors contribute to the errors of $\sigma$ and $r_{h}$. 


\begin{tabular}{l|c|c}
\hline Galaxy & $r_{h}$, Plummer & $r_{h}$, exponential \\
\hline Coma Berenices & $5.0^{\prime}$ & $5.9^{\prime}$ \\
\hline Canes Venatici II & $3.0^{\prime}$ & $3.3^{\prime}$ \\
\hline Leo IV & $3.3^{\prime}$ & $3.4^{\prime}$ \\
\hline Hercules & $8.0^{\prime}$ & $8.4^{\prime}$ \\
\hline
\end{tabular}

TABLE I: Uncertainties of determination of half-light radius $r_{h}$ for several dSphs.

\begin{tabular}{|c|c|c|c|c|c|c|c|c|}
\hline $\begin{array}{c}\mathrm{dSph} \\
(1)\end{array}$ & $\begin{array}{c}\text { References } \\
(2)\end{array}$ & $\begin{array}{l}r_{h} \\
\mathrm{pc} \\
(3) \\
\end{array}$ & $\begin{array}{c}\sigma \\
\mathrm{km} / \mathrm{s} \\
(4)\end{array}$ & $\begin{array}{c}\bar{F} \\
M_{\odot \mathrm{pc}^{-3}}(\mathrm{~km} / \mathrm{sec})^{-3} \\
(5)\end{array}$ & $\begin{array}{c}m_{\mathrm{DEG}} \\
\mathrm{keV} \\
(6)\end{array}$ & $\begin{array}{l}m_{\mathrm{FD}} \\
\mathrm{keV} \\
(7)\end{array}$ & $\begin{array}{c}m_{\mathrm{NRP}} \\
\mathrm{keV} \\
(8)\end{array}$ & $\begin{array}{c}m_{\mathrm{NRP}, \mathrm{TG}} \\
\mathrm{keV} \\
(9)\end{array}$ \\
\hline \multicolumn{9}{|c|}{ dSphs from 80] } \\
\hline Sextans & {$[80,113]$} & $630 \pm 170$ & $6.6 \pm 2.3$ & $4.78_{-2.58}^{+8.97} \cdot 10^{-7}$ & $0.147_{-0.026}^{+0.044}$ & $0.174_{-0.031}^{+0.053}$ & $0.454_{-0.104}^{+0.192}$ & $0.715_{-0.163}^{+0.302}$ \\
\hline Fornax & {$[80,113]$} & $400 \pm 103$ & $10.5 \pm 2.7$ & $7.45_{-3.70}^{+10.74} \cdot 10^{-7}$ & $0.164_{-0.026}^{+0.041}$ & $0.195_{-0.031}^{+0.049}$ & $0.527_{-0.108}^{+0.183}$ & $0.830_{-0.170}^{+0.288}$ \\
\hline Leo I & {$[80,113]$} & $330 \pm 106$ & $8.8 \pm 2.4$ & $1.31_{-0.72}^{+2.59} \cdot 10^{-6}$ & $0.189_{-0.034}^{+0.059}$ & $0.224_{-0.041}^{+0.070}$ & $0.635_{-0.148}^{+0.279}$ & $1.00_{-0.23}^{+0.44}$ \\
\hline UrsaMinor & {$[80,113]$} & $300 \pm 74$ & $9.3 \pm 2.8$ & $1.49_{-0.76}^{+2.27} \cdot 10^{-6}$ & $0.195_{-0.031}^{+0.051}$ & $0.232_{-0.037}^{+0.060}$ & $0.665_{-0.139}^{+0.240}$ & $1.05_{-0.22}^{+0.38}$ \\
\hline Carina & {$[80,113]$} & $290 \pm 72$ & $6.8 \pm 1.6$ & $2.19_{-1.05}^{+2.87} \cdot 10^{-6}$ & $0.215_{-0.032}^{+0.050}$ & $0.255_{-0.039}^{+0.060}$ & $0.755_{-0.148}^{+0.243}$ & $1.19_{-0.23}^{+0.38}$ \\
\hline Draco & {$[80,83]$} & $221 \pm 16$ & $9.5 \pm 1.6$ & $2.70_{-0.69}^{+1.07} \cdot 10^{-6}$ & $0.226_{-0.016}^{+0.020}$ & $0.269_{-0.019}^{+0.023}$ & $0.809_{-0.076}^{+0.095}$ & $1.27_{-0.12}^{+0.15}$ \\
\hline Bootes & {$[111,113,124]$} & $246 \pm 28$ & $6.5_{-1.3}^{+2.1}$ & $3.18_{-1.24}^{+1.88} \cdot 10^{-6}$ & $0.236_{-0.027}^{+0.029}$ & $0.280_{-0.033}^{+0.035}$ & $0.855_{-0.130}^{+0.143}$ & $1.35_{-0.20}^{+0.23}$ \\
\hline Sculptor & {$[80,113]$} & $160 \pm 40$ & $10.1 \pm 0.3$ & $4.99_{-1.98}^{+3.62} \cdot 10^{-6}$ & $0.264_{-0.031}^{+0.038}$ & $0.314_{-0.037}^{+0.046}$ & $0.993_{-0.154}^{+0.198}$ & $1.56_{-0.243}^{+0.312}$ \\
\hline Leo II & {$[80,113]$} & $185 \pm 48$ & $6.8 \pm 0.7$ & $5.38_{-2.30}^{+5.55} \cdot 10^{-6}$ & $0.269_{-0.035}^{+0.052}$ & $0.319_{-0.042}^{+0.062}$ & $1.02_{-0.17}^{+0.27}$ & $1.60_{-0.27}^{+0.43}$ \\
\hline \multicolumn{9}{|c|}{ dSphs from [79] } \\
\hline Canes Venatici I & {$[79,83]$} & $564 \pm 36$ & $7.6 \pm 2.2$ & $5.17_{-1.63}^{+3.46} \cdot 10^{-7}$ & $0.150_{-0.013}^{+0.020}$ & $0.178_{-0.016}^{+0.024}$ & $0.467_{-0.055}^{+0.087}$ & $0.735_{-0.087}^{+0.137}$ \\
\hline Ursa Major I & {$[79,83]$} & $318_{-39}^{+50}$ & $7.6 \pm 2.4$ & $1.63_{-0.70}^{+1.46} \cdot 10^{-6}$ & $0.199_{-0.026}^{+0.035}$ & $0.237_{-0.031}^{+0.041}$ & $0.684_{-0.118}^{+0.163}$ & $1.08_{-0.19}^{+0.26}$ \\
\hline Hercules & {$[79,83]$} & $330_{-52}^{+75}$ & $5.1 \pm 2.4$ & $2.25_{-1.28}^{+3.74} \cdot 10^{-6}$ & $0.216_{-0.041}^{+0.060}$ & $0.257_{-0.049}^{+0.071}$ & $0.762_{-0.187}^{+0.294}$ & $1.20_{-0.29}^{+0.46}$ \\
\hline Leo $\mathrm{T}$ & {$[79,83]$} & $178 \pm 39$ & $7.5 \pm 2.7$ & $5.26_{-2.66}^{+8.22} \cdot 10^{-6}$ & $0.267_{-0.043}^{+0.071}$ & $0.318_{-0.051}^{+0.084}$ & $1.01_{-0.21}^{+0.37}$ & $1.59_{-0.33}^{+0.59}$ \\
\hline Ursa Major II $^{a}$ & {$[79,83]$} & $140 \pm 25$ & $6.7 \pm 2.6$ & $9.53_{-4.59}^{+13.55} \cdot 10^{-6}$ & $0.310_{-0.047}^{+0.077}$ & $0.369_{-0.056}^{+0.091}$ & $1.23_{-0.24}^{+0.42}$ & $1.94_{-0.38}^{+0.67}$ \\
\hline Leo IV & {$[79,83]$} & $116_{-34}^{+26}$ & $3.3 \pm 2.8$ & $2.82_{-1.91}^{+34.39} \cdot 10^{-5}$ & $0.406_{-0.100}^{+0.368}$ & $0.483_{-0.119}^{+0.438}$ & $1.77_{-0.55}^{+2.41}$ & $2.79_{-0.87}^{+3.80}$ \\
\hline Coma Berenices & {$[79,83]$} & $77 \pm 10$ & $4.6 \pm 2.3$ & $4.59_{-2.19}^{+7.53} \cdot 10^{-5}$ & $0.459_{-0.069}^{+0.126}$ & $0.546_{-0.082}^{+0.150}$ & $2.08_{-0.41}^{+0.80}$ & $3.28_{-0.64}^{+1.25}$ \\
\hline Canes Venatici II & {$[79,83]$} & $74_{-10}^{+14}$ & $4.6 \pm 2.4$ & $4.97_{-2.66}^{+8.92} \cdot 10^{-5}$ & $0.468_{-0.082}^{+0.137}$ & $0.557_{-0.097}^{+0.163}$ & $2.14_{-0.48}^{+0.87}$ & $3.36_{-0.76}^{+1.38}$ \\
\hline
\end{tabular}

TABLE II: Parameters for dSphs from 79, 80] (columns 1-5) and derived lower mass limits for various types of DM (columns 6 9). $m_{\mathrm{DEG}}$ refers to the limit from Pauli exclusion principle (11), $m_{\mathrm{FD}}$ is the limit for particles with the momentum distribution (44), $m_{\mathrm{NRP}}$ and $m_{\mathrm{NRP}, \mathrm{TG}}-$ for distribution (20). All results are quoted for $g=2$ internal degrees of freedom. Results for NRP scenario are for $\omega_{\mathrm{DM}}=0.105[125]$.

${ }^{a}$ There is an extensive evidence that Ursa Major II is a tidally disrupted dSph [see e.g. 79]. Therefore, the results for it are provided for illustrative purposes only.

First of all, as $\sigma$ is the dispersion of measured velocities, it has the statistical error (which can be quite large for the ultra-faint dSphs where the number of stars can be rather small $(\sim 10-100$, c.f. [79, Table 3]). However, the systematic error is much larger. The authors of [79] found the systematic error on their determination of velocity dispersion to be $2.2 \mathrm{~km} / \mathrm{sec}$. We add this error in quadratures to the statistical errors, found in [79, Table 3]. The results are shown in the column number 4 in the Table I.

The half-light radius $r_{h}$ is a derived quantity and there are several contributions to its errors. First of all, the surface brightness profile is measured in angular units and their conversion to parsecs requires the knowledge of the distance towards the object. These distances are generally known with uncertainties of about 10\% (see 107, 110, 113, 113, 114, 115, 116, 117, 118, 119, 120, 121, 122, 123]). Another uncertainty comes from the method of determination of $r_{h}$. The surface brightness profile gets fit to various models to determine this quantity. For several dSphs: Coma Berenices, Canes Venatici II, Hercules and Leo IV authors used two different profiles (Plummer and exponential) for evaluating the annular half-light radius [107]. Their results are present in the Table【. We use these results to estimate the systematic error on $r_{h}$ to be $20 \%$ and use it for all the dSph, where $r_{h}$ is quoted without errors. The results of determination $r_{h}$ are shown in the 3rd column of the Table III. The obtained values of $\bar{F}$ with corresponding errors are presented in the Table II column 5. We determined the errors on $\bar{F}$ by pushing the uncertainties in both $\sigma$ and $r_{h}$ so that the values of $\bar{F}$ is minimized (maximized). 


\section{RESULTS}

Our main results are compiled into the Table II (columns 6-9). The column 6 of Table II contains the bound on $m_{\mathrm{DEG}}$ (given by Eq. (1)) based on the Pauli exclusion principle. It is independent of the details of the evolution of the system, is not affected by the presence of baryons (see below) and holds for any fermionic DM. The column 7 contains the mass bounds for the relativistically decoupled DM particles (primordial distribution (44)), obtained by combining Eqs.(17)-(19). Combining Eqs. (17), (18) and (23) one obtains the result for the case of DM with primordial velocity distribution (20), quoted in the column 8. Both bounds in columns 7 and 8 conservatively assume maximally coarse-grained distribution function (see Section III). In instead of the maximal coarse-graining, one assumes the isothermal distribution in the final state (c.f. Fig. 1), one arrives to the original Tremaine-Gunn bound, shown in the 9th column. It is obtained by comparing the expressions (21) with (24) ${ }^{9}$ We denote the corresponding mass bound by $m_{\mathrm{NRP}, \mathrm{TG}}$.

We quote all the mass bounds with the corresponding uncertainties, coming from those of in determination of $\sigma$ and $r_{h}$ (see Section [V). However, for any given object there can be unique reasons, violating the standard assumptions and therefore increasing the uncertainties. Therefore, although the strongest bounds in Table come from the Canes Venatici II (CVnII) dSph, we decided to take a value which independently follows from several objects as a single number, characterizing our results (for a given type of DM). To this end we choose the value, obtained for Leo IV. ${ }^{10}$ Thus, the mass bounds, quoted below are excluded from three dSphs: Leo IV, CVnII and Coma Berenices $(\text { Com })^{11}$ To summarize, we obtain the following lower bounds

$$
\begin{aligned}
& m_{\mathrm{DEG}}>0.41 \mathrm{keV}, \\
& m_{\mathrm{FD}}>0.48 \mathrm{keV}, \\
& m_{\mathrm{NRP}}>1.77 \mathrm{keV},
\end{aligned}
$$

and

$$
m_{\mathrm{NRP}, \mathrm{TG}}>2.79 \mathrm{keV} \text {. }
$$

We can compare lower bounds (34)- (35) with the upper ones, coming from astrophysical (X-ray) constraints on the possible flux from sterile neutrino DM decay [126, 127, 128, 129, 130, 131, 132, 133, 134, 135, 136]. Taking central value (34) and comparing it with the X-ray constraints, one sees that there exists a narrow window of parameters for which $100 \%$ of DM can be made from the NRP sterile neutrino (c.f. Fig 2). Less conservative bound (35), based on [22] (marked by the dark orange double-dotted vertical line on the Fig. 2) almost completely closes this window. Notice, that these bounds are comparable with the lower mass limit $m_{\mathrm{NRP}}>5.6 \mathrm{keV}$, coming from the Ly- $\alpha$ forest analysis of 74 .

We also performed the analysis for sterile neutrinos, produced in the presence of lepton asymmetry (resonant production mechanism, RP) 66, 67, 68]. This mechanism is more efficient than the NRP scenario and allows us to achieve the required DM abundance for weaker mixings (c.f. Fig. 4 in [68]). This lifts the upper bound on the DM particle mass in RP scenario to $\sim 50 \mathrm{keV}$. To estimate the lower mass bound at this scenario, we have analyzed a number of available spectra (mass range $1-20 \mathrm{keV}$, asymmetries $(2-700) \times 10^{-6}$ (see [67, 68] for the definition of asymmetry). The result are collected on the Fig. 3. One can see that based on $\bar{F}$, the $M_{\mathrm{RP}}=1 \mathrm{keV}$ is ruled out for lepton asymmetries $L \gtrsim 10^{2}$ and higher masses $M_{\mathrm{RP}} \geq 2 \mathrm{keV}$ are allowed for all available asymmetries. Based on the original Tremaine-Gunn bound, $M_{\mathrm{RP}}=2 \mathrm{keV}$ is also ruled out for sufficiently high $\left(L \gtrsim 10^{2}\right)$ lepton asymmetries. Thus, resonantly produced sterile neutrinos remain a viable DM candidate (see Fig. 4).

Finally, we would like to notice that our bounds (33)-35) are valid under the assumption that the influence of the baryons does not result in the increase of the PSD in the course of structure formation. If this assumption does not hold, only the bound (32) remains intact.

9 The value of $r_{c}$ is not currently known for several new, faint dSphs, from which we obtain the best limits on DM mass. Therefore, to calculate the Tremaine-Gunn limit in Table II we use the conservative estimate $r_{c} \approx r_{h}$ (see comment after Eq. (25)).

10 Notice, that the numbers for Leo IV essentially coincide with the mass limits from CVnII and Com if all uncertainties in these dSphs are pushed to minimize the mass bound.

${ }^{11}$ It is possible that Coma Berenices is undergoing tidal disruption (like another ultra-faint dSph, Ursa Major II (UMaII), closely resembling Com) [79]. However, unlike UMaII (or the best known example of tidally disrupted dSph, Sagittarius), there are no known tidal streams near the position of Coma Berenices and the evidence in favor of tidal disruption are quite moderate [c.f. discussion in 79, §3.6]. 


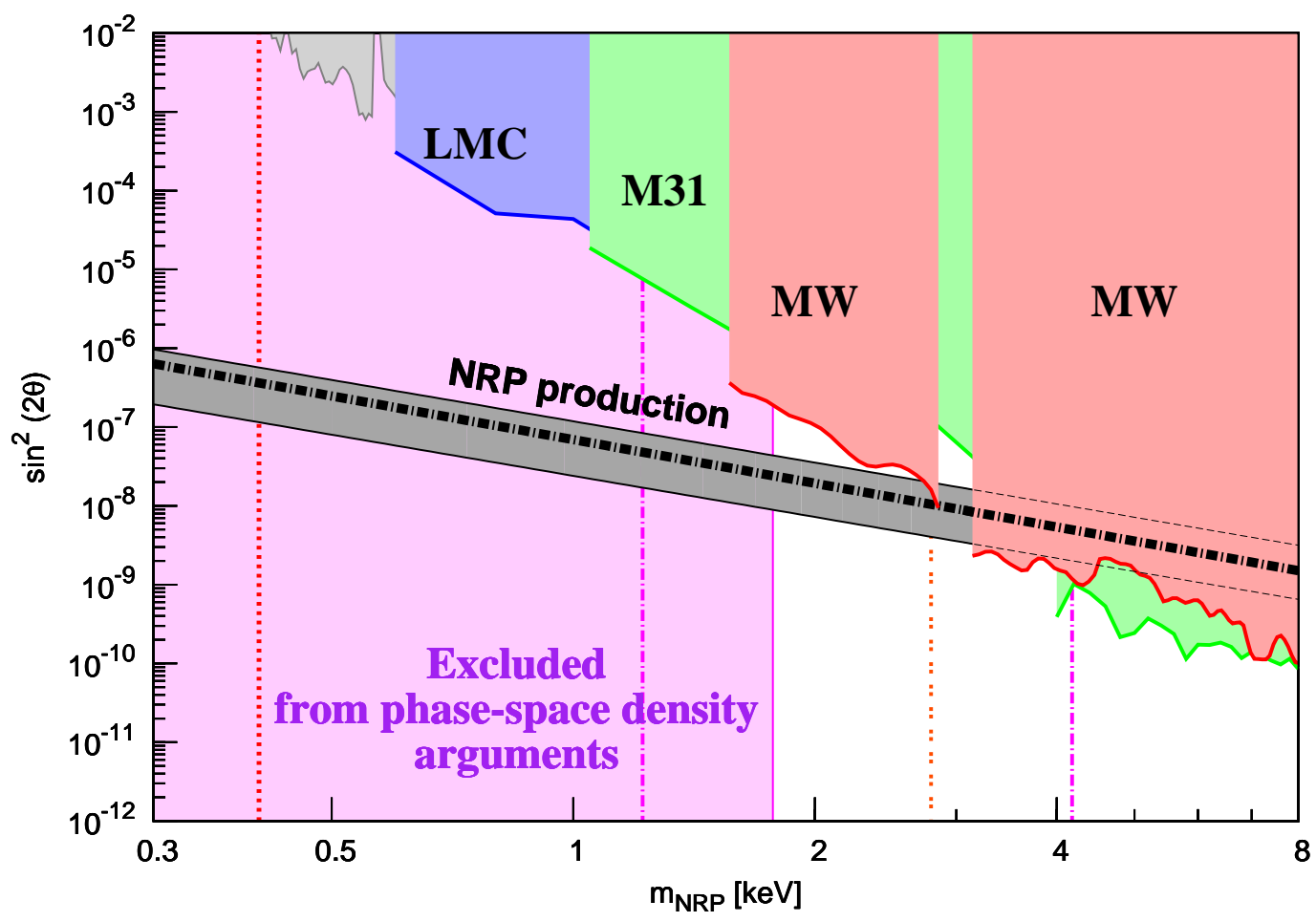

FIG. 2: Restrictions on parameters of sterile neutrino (mass and mixing $\sin ^{2}(2 \theta)$ between sterile and active neutrinos) from X-rays (128, 133, 134, 135, 136]) and phase-space density considerations (this work). Our analysis excludes the region to the left of the vertical line (34) (purple shaded region). Two dashed-dotted vertical lines mark the systematic uncertainties of this bound. The dotted line on the left marks the bound (32) based on the Pauli exclusion principle. The double-dotted dark orange line marks the bound (35). The black dashed-dotted line is the NRP production curve (i.e. pairs of $m_{\mathrm{NRP}}$ and $\theta$ that lead to the correct DM abundance) 65]. The gray region marked "NRP production" accounts for possible uncertainties in the abundance computations (see [64, 65] for details).

In this work we chose not to use the bound, based on the "average PSD" Q 26, 27] (see discussion in the Section ஹ). However, as this bound is widely used in the literature, we quote analogs of lower limits (33) and (334) based on inequality (9) (which we denote $m_{\mathrm{FD}, \mathrm{HD}}$ and $m_{\mathrm{NRP}, \mathrm{HD}}$ correspondingly):

$$
\begin{aligned}
m_{\mathrm{FD}, \mathrm{HD}} & =0.9 \mathrm{keV}, \\
m_{\mathrm{NRP}, \mathrm{HD}} & =4.0 \mathrm{keV} .
\end{aligned}
$$

For details see Appendix C,

\section{DISCUSSION}

In this paper we suggested that a conservative way to put the bound on a DM particle mass may be based on the requirements that the maximum of the observed coarse-grained phase space density should not exceed the maximum of the initial distribution function of the DM particles. The maximum of the coarse-graned distribution function in the final state may be conservatively estimated from the observed quantities. This bound relies on the assumption that the maximum of the distribution function was not significantly increased by the interaction with baryons.

Although DM consists of the non-interacting particles, the remaining part of the galaxy - the baryons - interact with one another and dissipate their energy, finally concentrating towards the center. The baryons, which are condensed in the center, influence the shape of DM halo gravitationally, increasing the central DM density [137, 138]. The opposite effect is the energy feedback from SNae, galactic winds and reionization, which creates the strong outflow, significantly decreasing the mass of the gas and thereby affecting the DM halo shape. Such a feedback is thought to be responsible to the formation of dwarf spheroidals from gas-rich dwarf spiral/irregular galaxies [139, 140, 141, 142, 143]. Clearly both gas condensation and feedback strongly influence the central PSD of DM [144], and in principle can lead to the 


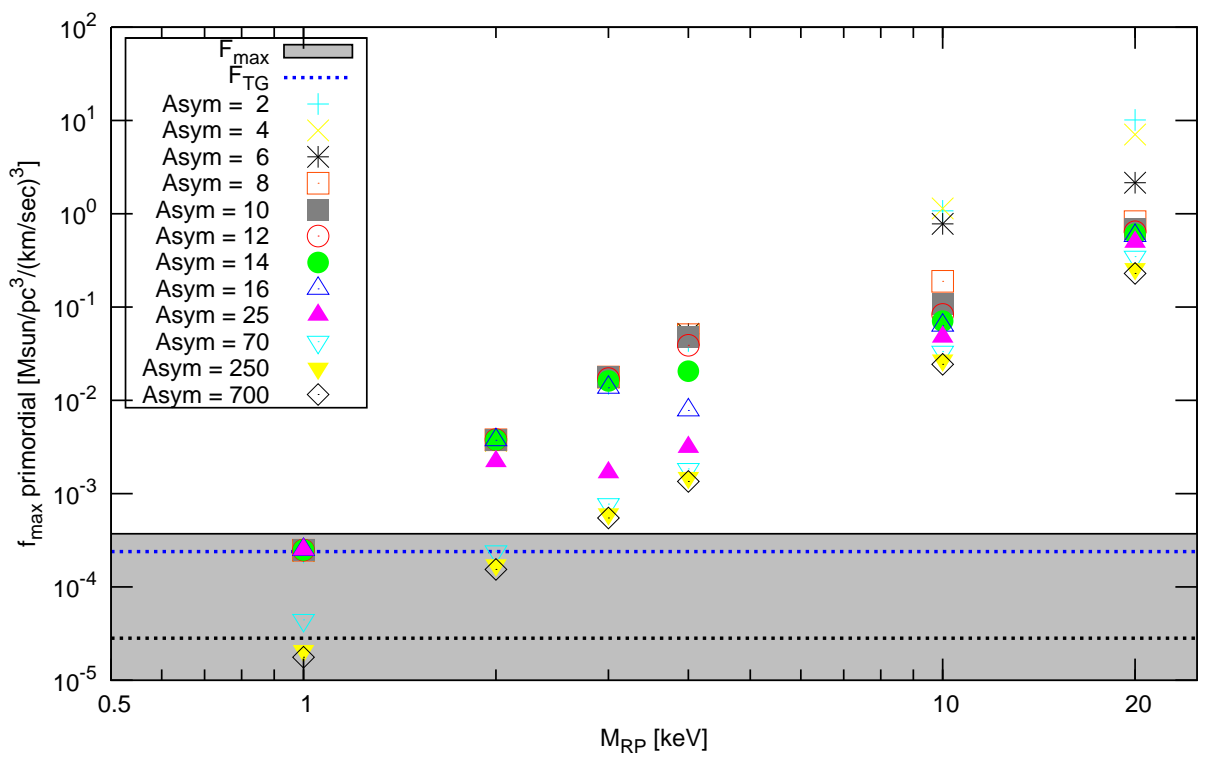

FIG. 3: Restrictions on resonantly produced sterile neutrinos. Primordial $f_{\max }$ is computed numerically based on the spectra from 67, 68]. Different colors parametrize different lepton asymmetries for a given mass (see definition of lepton asymmetry in [67, 68]). Grey shaded region is bounded by the maximal and minimal values of $\bar{F}$ for Leo IV (from Table [I] column 5). Horizontal dotted lines represent central value for $\bar{F}$ (lower) and $F_{\mathrm{TG}}$ (upper) for Leo IV. The DM spectrum is ruled out if the point falls into the shaded region (below the dotted line).

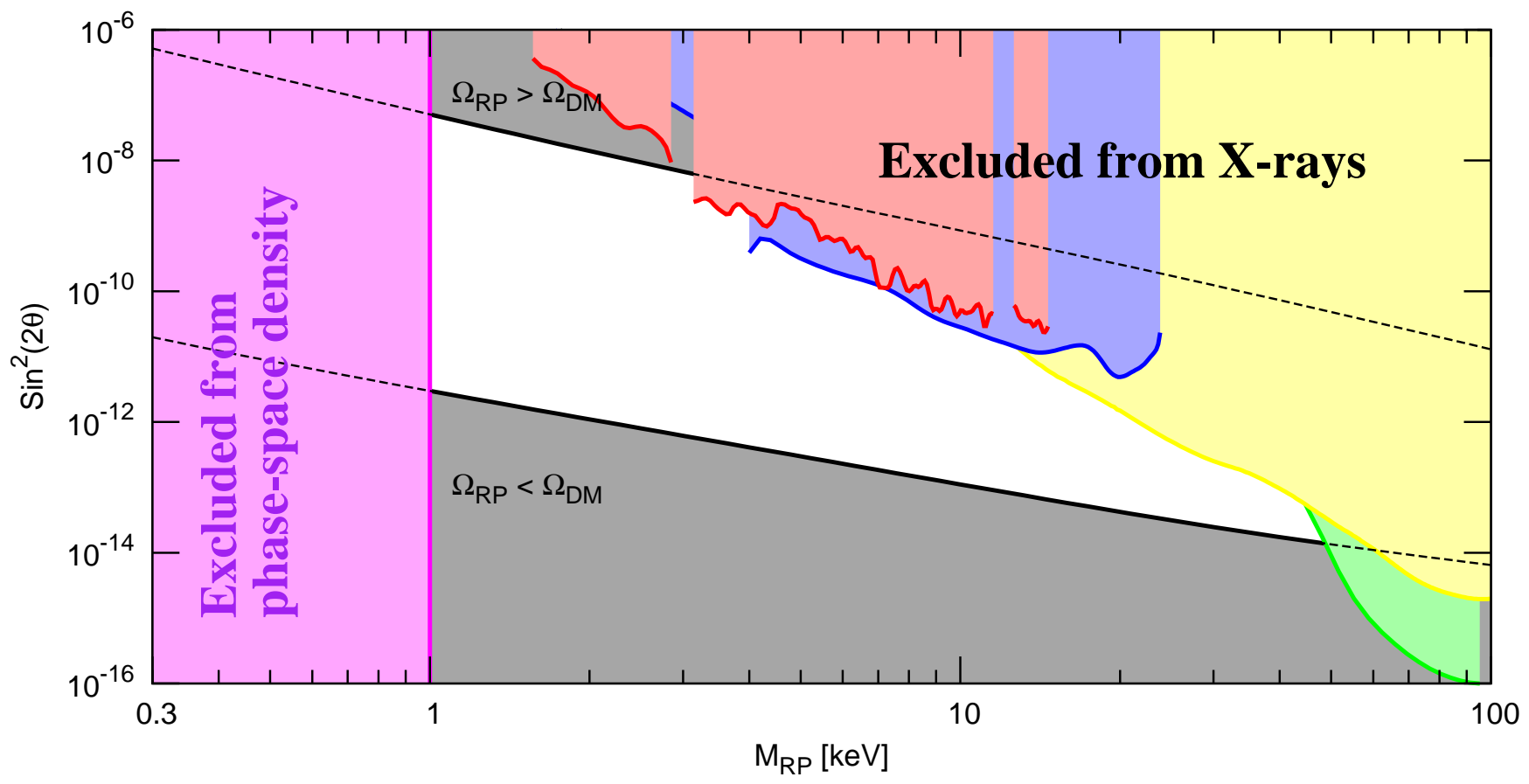

FIG. 4: Allowed window of parameters for sterile neutrinos produced via resonant oscillations (white unshaded strip between two black lines). Two bounding black lines are obtained for non-resonant (upper line, lepton asymmetry $=0$ ) and resonant production with the maximal lepton asymmetry, attainable in the $\nu$ MSM [67, 68] (lower line). The colored regions in the upper right corner represent X-ray bounds [128, 133, 135, 136]. Region below $1 \mathrm{keV}$ is ruled out from the PSD arguments (this work). 
violation of the inequality (3). Numerical studies of galaxy mergers show that baryons can lead to the increase of the phase-space density during the merger (see e.g. [145]). However, the method used in this work - coarse-graining of the PSD over a large phase-space region - reduces the influence of baryons. Indeed, we take the spatial averaging over the radius $R \sim r_{h}$, which includes external part of the system, where the amount of baryons is small. Additional studies are necessary to estimate effects of baryons and make our bounds more robust. We plan to address these issues elsewhere.

We would also like to stress that the initial velocities of DM particles in our approach are thermal velocities and they should not be confused with the so-called Zeldovich velocities [2]. Numerical simulations of galaxy formation do not start at the time, when the DM phase-space distribution is spatially uniform (redshifts $z \gtrsim 10^{3}$ ). Instead, the initial (linear) stage of the structure formation is computed analytically in the framework of the so-called Zeldovich approximation [2]. This approximation is commonly used to set up initial conditions for the numerical simulations of non-linear stage of structure formation [146, 147, 148], which start at redshifts $z \sim 10$. The peculiar (Zeldovich) velocities acquired by DM particles at this stage due to structure formation and included into the initial conditions are normally $\sigma \sim 10 \mathrm{~km} /$ sec. Apart from Zeldovich velocities, DM particles also possess thermal velocities, which are discussed in this paper. For cold enough Dark Matter these thermal velocities are much smaller than Zeldovich ones and, thus, are often neglected and not included into initial conditions. Therefore, the numerical studies of PSD evolution $^{12}$ (see e.g. 149, 150, 151, 152, 153, 154]) essentially investigate the change of PSD from Zeldovich to final stage. It was found in some of these works that the PSD changes by $10^{2}-10^{3}$ in the process of collapse [150]. This change of PSD can be understood as being simply an evolution from initial Zeldovich velocities $\sigma_{i} \sim 10 \mathrm{~km} / \mathrm{sec}$ to the final (virial) ones $\sigma_{f} \sim 10^{2} \mathrm{~km} / \mathrm{sec}$ (with $Q_{i} / Q_{f} \sim\left(\sigma_{f} / \sigma_{i}\right)^{3} \sim 10^{3}$ ).

Because initial thermal velocities may be much smaller than Zeldovich ones, initial PSD may differ from the final (observed) PSD not by 2-3, but by many orders of magnitude. This fact does not contradict to the results of simulations, described in e.g. [150] and, therefore, cannot be used to obtain an upper bound on the mass of DM particles (c.f [88, 155]).

This work was mostly concentrated on restrictions on the mass of the sterile neutrino DM, produced in through the non-resonant oscillations with active neutrino (NRP scenario). We see that our results (Section V) strongly disfavor such sterile neutrinos as the single DM component. This conclusion is not based on the Ly- $\alpha$ method and therefore is not subject to its uncertainties (discussed in the Introduction). However, several uncertainties can affect this conclusion, the major being baryonic feedback. To make this result really robust, apart from further modeling of the baryonic influence, one needs to strengthen the tension between upper and lower mass bounds discussed in this paper. This is plausible and may be done either by improving the X-ray bounds with new observations or by strengthening the PSD consideration, which is in the first place related to better measurements of kinematics of dSphs.

In the presence of lepton asymmetry, the resonant production (RP) of sterile neutrino DM takes place 66. This mechanism is more efficient 66, 67, 68] than the NRP scenario and allows to achieve required DM abundance for weaker mixings (c.f. Fig. 4 in [68]). This lifts the upper bound on the DM particle mass in this scenario up to $\sim 50 \mathrm{keV}$. At the same time, for the same mass the primordial velocity distribution of RP sterile neutrino DM is colder than in NRP one. This $f_{\max }$ is as much as the order of magnitude bigger than (21) (c.f. [68]). This brings down by a factor $\sim 2$ the analog of the mass bound (34). Analyzing available spectra for a range of lepton asymmetries, we see that models with $m_{\mathrm{RP}} \gtrsim 1 \mathrm{keV}$ are allowed. Thus, there is a large open "window" of allowed DM masses (c.f. Fig (4). However, as the dependence of the velocity spectrum on the lepton assymetry is not monotonic, to obtain the exact shape of the lower bound on the mass at given mixing angle more work is needed. Nevertheless, our results show that the sterile neutrinos, produced in the presence of lepton asymmetry, are viable DM candidates, allowed by all current bounds.

Finally, we would like to comment on the mechanism of production of sterile neutrinos from decay of massive scalar field, for example the inflaton [69] (for other models see [57, 70, 71, 155]). The primordial phase-space distribution function for this case was computed e.g. in [69, 70, 155, 156]. Maximal value of phase-space density for this distribution is that of degenerate Fermi gas. Notice that the distribution functions in [69, 70, 155] $f(p)$ is formally unbounded for small momenta: $f(p) \sim p^{-1 / 2}$. From this one can easily find that the fraction of particles, having maximal phase-space density, is $\sim 10^{-8}$. As only this small fraction of all particles has maximal phase-space density, we expect the mass bound in this case to be stronger than (32). The detailed analysis will be presented elsewhere.

After this work has been completed, we received a draft of the paper [157], where similar issues have been considered. Our results are consistent with those of discussed in [157] wherever they overlap.

12 Most of these studies use the quantity $Q(r)=\rho(r) / \sigma^{3}(r)$ as a PSD estimator 


\section{Acknowledgments}

We would like to thank F. Bezrukov, G. Gilmore, D. Gorbunov, C. Hogan, A. Macciò, B. Moore, T. Naab, V. Rubakov, M. Shaposhnikov, T. Theuns, I. Tkachev, M. Viel for useful comments. We are grateful to M. Laine for providing to us the distribution functions for the RP sterile neutrinos. D.I. is grateful to to Scientific and Educational Centre $^{13}$ of the Bogolyubov Institute for Theoretical Physics in Kiev, Ukraine, and especially to V. Shadura, for creating wonderful atmosphere for young Ukrainian scientists. This work was supported by the Swiss National Science Foundation and the Swiss Agency for Development and Cooperation in the framework of the programme SCOPES - Scientific co-operation between Eastern Europe and Switzerland. D.I. also acknowledges support from the "Cosmomicrophysics" programme and from the Program of Fundamental Research of the Physics and Astronomy Division of the National Academy of Sciences of Ukraine. O.R. would like to acknowledge support of the Swiss National Science Foundation.

\section{APPENDIX A: INFLUENCE OF ASPHERICAL SHAPES OF DM HALOS}

We analyze the change of the bound eq. (1) due to the deviation of a DM halo from a spherical shape. Such asphericity affects both the spatial volume $V$ and the escape velocity $v_{\infty}$. We consider the dSph as homogeneous ellipsoid with semi-axes $a, b$ and $c$ and assume the ellipticity of its $2 \mathrm{D}$ projection ${ }^{14} \epsilon \lesssim 0.5$. Because we observe only 2D projection of such an ellipsoid, there are two possibilities:

Prolate dSph: $c>b \simeq a$. We see the axes $b$ and $c$, related to the "averaged" radius $R$ via $b=R(1-\epsilon)^{1 / 2}, c=$ $R(1-\epsilon)^{-1 / 2}$. The spatial volume $V$ is therefore

$$
V=\frac{4}{3} \pi a b c \approx \frac{4}{3} \pi R^{3}(1-\epsilon)^{1 / 2} \approx \frac{4}{3} \pi R^{3}(1-0.5 \epsilon) .
$$

The gravitational potential for $\epsilon \lesssim 0.5$ is dominated by monopole and quadrupole components,

$$
\phi \approx \phi^{(0)}+\phi^{(2)} .
$$

The maximal value of the potential occurs near the end of the minor semi-axis:

$$
\left|\phi_{\max }\right| \equiv \frac{v_{\infty}^{2}}{2}=\frac{G_{N} M}{a}-\frac{G_{N} D_{z z}}{4 a^{3}},
$$

where $D_{z z}=\frac{2 M\left(c^{2}-a^{2}\right)}{5}-$ the quadrupole moment of the system [158]. For $\epsilon \ll 1$ we then obtain

$$
\frac{\left.V v_{\infty}^{3}\right|_{\text {prolate }}}{\left.V v_{\infty}^{3}\right|_{\text {spherical }}} \approx 1+0.05 \epsilon
$$

which gives us the correction for $m_{\mathrm{DEG}}$ of smaller than $1 \%$ (for $\epsilon=0.5$ ).

Oblate dSph: $c \simeq b>a$. We observe the axes $a$ and $c$, therefore the spatial volume $V$ changes by $(1-\epsilon)^{-1 / 2} \approx$ $1+0.5 \epsilon$. The maximum of the gravitational potential is then given by

$$
\left|\phi_{\max }\right| \approx \frac{G_{N} M}{a}+\frac{G_{N} D_{x x}}{2 a^{3}} \approx \frac{G_{N} M}{R}(1+0.1 \epsilon) .
$$

where $D_{x x}$ is given by the same expression, as $D_{z z}$ above. The maximal phase-space volume changes in the oblate case by $\approx 1+0.65 \epsilon$, so the correction for $m_{\mathrm{DEG}}$ will constitute about $8 \%$ for $\epsilon \simeq 0.5$.

Thus, the departure from spherical symmetry for DM halos of dSphs changes the limit on $m_{\mathrm{DEG}}$ by less than $\lesssim$ $10 \%$ for the case of axis ratio 1:2. This uncertainty is below several others, therefore, we will consider dSphs to be spherical in what follows.

13 http://sec.bitp.kiev.ua

14 Throughout this paper, we define the ellipticity $\epsilon$ in a way similar to that in [89] (see also [83]), i.e. $\epsilon \equiv 1-b / a$, where $a$ and $b$ are the semi-major and semi-minor axis, respectively. Thus, the case of $\epsilon=0.5$ corresponds to axis ratio $1: 2$. 


\section{APPENDIX B: ENTROPY FOR DIFFERENT DISTRIBUTIONS}

In this Appendix we will calculate the entropy for several phase-space distributions, including those of (4), (20), (5), and explore its relation with the quantity $Q$, defined in (7).

The entropy of an ideal Fermi gas is given by the expression [159]

$$
S=-\int d^{3} p d^{3} r\left[f(r, p) \log \left(\frac{(2 \pi \hbar)^{3} f(r, p)}{g}\right)+\left(\frac{g}{(2 \pi \hbar)^{3}}-f(r, p)\right) \log \left(1-\frac{(2 \pi \hbar)^{3} f(r, p)}{g}\right)\right] .
$$

If the distribution function $f(r, p) \ll \frac{g}{(2 \pi \hbar)^{3}}$, we obtain the expression for the entropy of a non-degenerate ideal gas:

$$
S=-\int d^{3} p d^{3} r f(r, p)\left[\log \left(\frac{(2 \pi \hbar)^{3} f(r, p)}{g}\right)-1\right] .
$$

\section{Ideal Boltzmann gas}

We start with the case of ideal Boltzmann gas:

$$
f(r, p)=f_{0} e^{-\frac{p^{2}}{2 m T}} .
$$

Substituting it into Eq. (B2), we arrive to the well-known expression (c.f. e.g. [159, §42]):

$$
\frac{S}{N}=\frac{5}{2}+\log \left(\frac{g V}{N} \frac{(m T)^{3 / 2}}{\left(2 \pi \hbar^{2}\right)^{3 / 2}}\right)
$$

where $V$ is the volume of the system, $N$ is a number of particles. Expressing $S / N$ as a function of $\bar{\rho}$ and $\left\langle v^{2}\right\rangle$, we finally obtain relation between the entropy and Qin the form (10)

$$
\frac{S}{N}=\log C_{B}-\log \frac{Q \hbar^{3}}{m^{4}}, C_{B}=g \frac{e^{5 / 2}}{(6 \pi)^{3 / 2}} \approx g \times 0.1489 \ldots
$$

\section{Isothermal phase-space density distrubution}

Next, we consider the case when the PSD distribution can be approximated by (pseudo)-isothermal sphere (c.f. (5D)):

$$
f_{i s o}(r, p)=\frac{9 \sigma^{2}}{4 \pi G_{N}\left(2 \pi m^{2} \sigma^{2}\right)^{3 / 2}\left(r^{2}+r_{c}^{2}\right)} e^{-\frac{p^{2}}{2 m^{2} \sigma^{2}}} .
$$

The number of particles in such a system, as well as the total entropy, diverges for large $r$, however the entropy per particles grows logarithmically at large $r$ and therefore the exact value of cut-off is not important.

Truncating the expression for the entropy at some $r_{\max }$ and taking $r_{\max } \gg r_{c}$, we obtain

$$
\frac{S}{N}=-\log \frac{Q \hbar^{3}}{m^{4}}+\log C_{\text {iso }}, \quad C_{\text {iso }}=\frac{g \exp (1 / 2)}{\sqrt{3}(2 \pi)^{3 / 2}} \approx g \times 0.0604 \ldots
$$

\section{Entropy for Fermi-Dirac and NRP distributions}

Next, we analyze the case of primordial momentum distribution, which has the form of (rescaled) relativistic Fermi-Dirac.

$$
f(p)=\frac{g}{(2 \pi \hbar)^{3}} \frac{F}{e^{\epsilon(p) / T}+1}, \quad \epsilon(p)=p .
$$

For now we keep both $F$ and $T$ to be arbitrary. The distribution in the form (B8) accounts for both (44) and (20) cases. The entropy of $N$ particles with distribution (B8) is given by the expression (B1), which reduces to

$$
S=\frac{g V T^{3}}{2 \pi^{2} \hbar^{3}} I(F),
$$


where function $I(F)$ is given by

$$
I(F) \equiv-\int_{0}^{\infty} d z z^{2}\left[\frac{F}{e^{z}+1} \log \left(\frac{F}{e^{z}+1}\right)+\left(1-\frac{F}{e^{z}+1}\right) \log \left(1-\frac{F}{e^{z}+1}\right)\right] .
$$

The integral (B10) can be computed numerically. At $F \ll 1$ the expression (B10) can be approximated by

$$
I(F) \approx \frac{3}{2} \zeta(3)(F-F \log F)+F \int_{0}^{\infty} \frac{d z z^{2}}{e^{z}+1} \log \left(e^{z}+1\right) .
$$

The specific entropy $S / N$ equals to

$$
\frac{S}{N}=\frac{g m^{4} I(F)}{2 \pi^{2} \hbar^{3}}\left(\frac{\zeta(3)}{15 \zeta(5)}\right)^{3 / 2} \frac{\left\langle v^{2}\right\rangle^{3 / 2}}{\bar{\rho}}=\frac{g I(F)}{2 \pi^{2}}\left(\frac{\zeta(3)}{15 \zeta(5)}\right)^{3 / 2} \frac{m^{4}}{Q \hbar^{3}} .
$$

Therefore, we see that for the distributions of the form (B8) relation between the entropy per particle and $Q$ is not given by the simple expression (10).

Up until this moment we kept parameters $F$ and $T$ in (B8) independent. However, we are mostly interested in two particular cases: (i) $F=1$ while $T=T_{\mathrm{FD}}$ - arbitrary (distribution (44)); and (ii) $F<1$ having arbitrary value, while $T$ being fixed to $T_{\nu}$ - the temperature of neutrino background, related to the temperature of the CMB background today via $T_{\nu_{0}}=(4 / 11)^{1 / 3} T_{\mathrm{CMB}, 0}$ (distribution (20) ).

We start with the case (i). Expressing $\rho$ as a function of $T_{\mathrm{FD}}$, we obtain

$$
Q=\frac{g m^{4}}{\hbar^{3}} \mathbf{q}
$$

where numerical constant $\mathbf{q}$ is given by (c.f. [27]):

$$
\mathbf{q}=\frac{\zeta^{5 / 2}(3)}{20 \pi^{2} \sqrt{15} \zeta^{3 / 2}(5)} \approx 1.96 \ldots \times 10^{-3} .
$$

As a result for the distribution (4) and fixed number of particles, the quantity $Q$ is independent on $T_{\mathrm{FD}}$, volume or $N$. The entropy per particle is also independent on both $T_{\mathrm{FD}}$ and $V$ and is given by

$$
\frac{S}{N}=\mathbf{s}=I(1) \frac{2}{3 \zeta(3)} \approx 4.20 \ldots
$$

Although both quantities $S / N$ and $Q$ are simply constants, we find it convenient to choose them in the form (10):

$$
\frac{S}{N}=-\log \left(\frac{Q \hbar^{3}}{m^{4}}\right)+\log C_{\mathrm{FD}}, \quad C_{\mathrm{FD}}=g \cdot \mathbf{q} \cdot e^{\mathbf{s}} \approx g \times 0.1311 \ldots
$$

In case (ii) when $F \ll 1$ we obtain for $S / N$ :

$$
\frac{S}{N}=\frac{2}{3 \zeta(3)} \frac{I(F)}{F} \simeq(1-\log F)+\frac{2 l}{3 \zeta(3)} .
$$

Similarly to (B13) $-(\overline{\mathrm{B} 14})$

$$
\frac{Q \hbar^{3}}{m^{4}}=g \mathbf{q} F
$$

Combining (B17) $-(\overline{B 18})$ we can write

$$
\frac{S}{N}=-\log \left(\frac{Q \hbar^{3}}{m^{4}}\right)+\log C_{\mathrm{NRP}}, \quad C_{\mathrm{NRP}}=g \mathbf{q} \exp \left(1+\frac{2 l}{3 \zeta(3)}\right) \approx g \times 0.137 \ldots
$$




\begin{tabular}{|l|c|c|c|}
\hline \multicolumn{1}{|c|}{ dSph } & $\begin{array}{c}Q_{f} \\
{\left[\frac{M_{\odot}}{\mathrm{pc}^{3}}\left(\frac{\mathrm{km}}{\mathrm{sec}}\right)^{-3}\right.}\end{array}$ & $\begin{array}{c}m_{\mathrm{FD}, \mathrm{HD}} \\
{[\mathrm{keV}]}\end{array}$ & $\begin{array}{c}m_{\mathrm{NRP}, \mathrm{HD}} \\
{[\mathrm{keV}]}\end{array}$ \\
\hline Sextans & $5.68_{-3.07}^{+10.67} \cdot 10^{-6}$ & $0.324_{-0.057}^{+0.098}$ & $1.04_{-0.24}^{+0.44}$ \\
Fornax & $8.86_{-4.40}^{+12.77} \cdot 10^{-6}$ & $0.362_{-0.051}^{+0.091}$ & $1.20_{-0.25}^{+0.42}$ \\
Leo I & $1.55_{-0.85}^{+3.08} \cdot 10^{-5}$ & $0.416_{-0.075}^{+0.131}$ & $1.45_{-0.34}^{+0.64}$ \\
UrsaMinor & $1.78_{-0.90}^{+2.70} \cdot 10^{-5}$ & $0.431_{-0.070}^{+0.112}$ & $1.52_{-0.32}^{+0.55}$ \\
Bootes & $3.78_{-1.48}^{+2.24} \cdot 10^{-5}$ & $0.520_{-0.061}^{+0.064}$ & $1.95_{-0.30}^{+0.33}$ \\
Draco & $3.21_{-0.82}^{+1.27} \cdot 10^{-5}$ & $0.499_{-0.036}^{+0.044}$ & $1.85_{-0.17}^{+0.22}$ \\
Carina & $2.60_{-1.25}^{+3.42} \cdot 10^{-5}$ & $0.474_{-0.072}^{+0.111}$ & $1.72_{-0.34}^{+0.56}$ \\
Sculptor & $5.93_{-2.35}^{+4.30} \cdot 10^{-5}$ & $0.582_{-0.069}^{+0.085}$ & $2.27_{-0.35}^{+0.45}$ \\
Leo II & $6.39_{-2.73}^{+6.60} \cdot 10^{-5}$ & $0.593_{-0.077}^{+0.115}$ & $2.32_{-0.40}^{+0.62}$ \\
Canes Venatici I & $6.16_{-1.94}^{+4.11} \cdot 10^{-6}$ & $0.330_{-0.030}^{+0.045}$ & $1.07_{-0.13}^{+0.20}$ \\
Ursa Major I & $1.94_{-0.84}^{+1.74} \cdot 10^{-5}$ & $0.440_{-0.058}^{+0.077}$ & $1.56_{-0.27}^{+0.37}$ \\
Hercules & $2.68_{-1.53}^{+4.45} \cdot 10^{-5}$ & $0.477_{-0.091}^{+0.132}$ & $1.74_{-0.43}^{+0.67}$ \\
Leo T & $6.26_{-3.16}^{+9.78} \cdot 10^{-5}$ & $0.590_{-0.095}^{+0.157}$ & $2.31_{-0.48}^{+0.85}$ \\
Ursa Major II ${ }^{a}$ & $1.13_{-0.55}^{+1.61} \cdot 10^{-4}$ & $0.685_{-0.104}^{+0.169}$ & $2.81_{-0.55}^{+0.96}$ \\
Leo IV & $3.35_{-2.27}^{+40.91} \cdot 10^{-4}$ & $0.898_{-0.221}^{+0.814}$ & $4.04_{-1.27}^{+5.51}$ \\
Canes Venatici II & $5.91_{-3.16}^{+10.61} \cdot 10^{-4}$ & $1.03_{-0.18}^{+0.30}$ & $4.88_{-1.10}^{+1.99}$ \\
Coma Berenices & $5.46_{-2.61}^{+8.96} \cdot 10^{-4}$ & $1.01_{-0.15}^{+0.28}$ & $4.75_{-0.92}^{+1.82}$ \\
\hline
\end{tabular}

TABLE III: The mass bounds, based on the evolution of the average PSD $Q$ [26, 27]. The bound is provided for illustration purposes only (see Section $\mathrm{C}$ for discussion).

\section{APPENDIX C: MASS BOUNDS FROM THE EVOLUTION OF THE AVERAGE PSD}

For illustration purposes we provide in Table III the average PSD estimator $Q$ for all the dSphs, considered in this work, as well as the lower mass bounds, based on the inequality (9) for $Q$ during the evolution [26, 27] (for detailed discussion see Section (II). The value of $Q_{f}$, shown in the second column of the Table $\amalg$ in calculated from the data in the columns (3-4) of the Table II], using formula (26) (with $\eta=1$ ) and $Q_{i}$ is defined via (8) for the momentum distributions (4) and (20) (for the bounds $m_{\mathrm{FD}, \mathrm{HD}}$ and $m_{\mathrm{NRP}, \mathrm{HD}}$ correspondingly). The results for Leo IV are quoted in (36) (Section V).

[1] S. Calchi Novati (2007), 0711.4474.

[2] Y. B. Zel'dovich, A\&A 5, 84 (1970).

[3] G. S. Bisnovatyi-Kogan, AZh 57, 899 (1980).

[4] J. R. Bond, G. Efstathiou, and J. Silk, Phys. Rev. Lett. 45, 1980 (1980).

[5] A. G. Doroshkevich, M. I. Khlopov, R. A. Sunyaev, A. S. Szalay, and I. B. Zeldovich, New York Academy Sciences Annals 375, 32 (1981).

[6] J. R. Bond and A. S. Szalay, ApJ 274, 443 (1983).

[7] S. D. M. White, C. S. Frenk, and M. Davis, ApJ 274, L1 (1983).

[8] P. J. E. Peebles, Science 224, 1385 (1984).

[9] L. Bergstrom, Rept.Prog.Phys. 63, 793 (2000), hep-ph/0002126.

[10] J. Carr, G. Lamanna, and J. Lavalle, Reports of Progress in Physics 69, 2475 (2006).

[11] M. Taoso, G. Bertone, and A. Masiero, JCAP 0803, 022 (2008), 0711.4996.

[12] G. Bertone, D. Hooper, and J. Silk, Phys. Rep. 405, 279 (2005), arXiv:hep-ph/0404175.

[13] S. L. Dubovsky, P. G. Tinyakov, and I. I. Tkachev, Phys. Rev. Lett. 94, 181102 (2005), hep-th/0411158.

[14] R. Holman, G. Lazarides, and Q. Shafi, Phys. Rev. D 27, 995 (1983).

[15] S. Dodelson and L. M. Widrow, Phys. Rev. Lett. 72, 17 (1994), hep-ph/9303287.

[16] H. Pagels and J. R. Primack, Phys. Rev. Lett. 48, 223 (1982).

[17] H. E. Haber and G. L. Kane, Phys. Rep. 117, 75 (1985).

[18] L. Covi, J. E. Kim, and L. Roszkowski, Phys. Rev. Lett. 82, 4180 (1999), arXiv:hep-ph/9905212.

[19] A. Kusenko and M. E. Shaposhnikov, Phys. Lett. B418, 46 (1998), hep-ph/9709492.

[20] V. A. Kuzmin and I. I. Tkachev, JETP Lett. 68, 271 (1998), hep-ph/9802304.

[21] D. J. H. Chung, E. W. Kolb, and A. Riotto, Phys. Rev. D 59, 023501 (1999), arXiv:hep-ph/9802238. 
[22] S. Tremaine and J. E. Gunn, Phys. Rev. Lett. 42, 407 (1979).

[23] J. Madsen and R. I. Epstein, ApJ 282, 11 (1984).

[24] J. Madsen, Phys. Rev. D 44, 999 (1991).

[25] J. Madsen, Physical Review Letters 64, 2744 (1990).

[26] J. J. Dalcanton and C. J. Hogan, ApJ 561, 35 (2001), astro-ph/0004381.

[27] C. J. Hogan and J. J. Dalcanton, Phys. Rev. D62, 063511 (2000), astro-ph/0002330.

[28] J. Madsen, Phys. Rev. D 64, 027301 (2001), arXiv:astro-ph/0006074.

[29] L. Hui, N. Y. Gnedin, and Y. Zhang, ApJ 486, 599 (1997), astro-ph/9608157.

[30] N. Y. Gnedin and A. J. S. Hamilton, Mon.Not.Roy.Astron.Soc. 334, 107 (2002), astro-ph/0111194.

[31] D. H. Weinberg, R. Davé, N. Katz, and J. A. Kollmeier, in AIP Conf. Proc. 666: The Emergence of Cosmic Structure, edited by S. H. Holt and C. S. Reynolds (2003), pp. 157-169.

[32] A. Lewis and S. Bridle, Phys. Rev. D66, 103511 (2002), astro-ph/0205436.

[33] T. Theuns, A. Leonard, G. Efstathiou, F. R. Pearce, and P. A. Thomas, Mon. Not. Roy. Astron. Soc. 301, 478 (1998), astro-ph/9805119.

[34] P. McDonald, U. Seljak, S. Burles, D. J. Schlegel, D. H. Weinberg, R. Cen, D. Shih, J. Schaye, D. P. Schneider, N. A. Bahcall, et al., ApJS 163, 80 (2006), arXiv:astro-ph/0405013.

[35] M. Viel, M. G. Haehnelt, and V. Springel, Mon. Not. Roy. Astron. Soc. 354, 684 (2004), astro-ph/0404600.

[36] M. Viel, J. Lesgourgues, M. G. Haehnelt, S. Matarrese, and A. Riotto, Phys. Rev. D71, 063534 (2005), astro-ph/0501562.

[37] M. Viel, M. G. Haehnelt, and V. Springel, Mon.Not.Roy.Astron.Soc. 367, 1655 (2006), astro-ph/0504641.

[38] M. Viel and M. G. Haehnelt, Mon. Not. Roy. Astron. Soc. 365, 231 (2006), astro-ph/0508177.

[39] J. A. Regan, M. G. Haehnelt, and M. Viel, Mon. Not. Roy. Astron. Soc. 374, 196 (2007), astro-ph/0606638.

[40] T. S. Kim, J. S. Bolton, M. Viel, M. G. Haehnelt, and R. F. Carswell (2007), 0711.1862.

[41] J. S. Bolton, M. Viel, T. S. Kim, M. G. Haehnelt, and R. F. Carswell (2007), 0711.2064.

[42] M. Viel, S. Matarrese, H. J. Mo, T. Theuns, and M. G. Haehnelt, Mon. Not. Roy. Astron. Soc. 336, 685 (2002), astro$\mathrm{ph} / 0203418$.

[43] M. Viel, S. Matarrese, H. J. Mo, M. G. Haehnelt, and T. Theuns, Mon. Not. Roy. Astron. Soc. 329, 848 (2002), astro$\mathrm{ph} / 0105233$.

[44] M. Viel, M. G. Haehnelt, R. F. Carswell, and T. S. Kim, Mon. Not. Roy. Astron. Soc. 349, L33 (2004), astro-ph/0308078.

[45] P. L. Biermann and A. Kusenko, Phys. Rev. Lett. 96, 091301 (2006), astro-ph/0601004.

[46] L. Gao and T. Theuns, Science 317, 1527 (2007), arXiv:0709.2165.

[47] J. Stasielak, P. L. Biermann, and A. Kusenko, ApJ 654, 290 (2007), arXiv:astro-ph/0606435.

[48] A. Boyarsky, J. Lesgourgues, O. Ruchayskiy, and M. Viel (2008), 0812.0010.

[49] T. Asaka, S. Blanchet, and M. Shaposhnikov, Phys. Lett. B631, 151 (2005), hep-ph/0503065.

[50] G. L. Fogli, E. Lisi, A. Marrone, A. Palazzo, and A. M. Rotunno, Prog. Part. Nucl. Phys. 57, 71 (2006), arXiv:hep$\mathrm{ph} / 0506083$.

[51] A. Strumia and F. Vissani (2006), hep-ph/0606054.

[52] C. Giunti, Nucl. Phys. Proc. Suppl. 169, 309 (2007), hep-ph/0611125.

[53] T. Asaka and M. Shaposhnikov, Phys. Lett. B620, 17 (2005), hep-ph/0505013.

[54] A. D. Dolgov (1997), hep-ph/9707419.

[55] A. Riotto (1998), hep-ph/9807454.

[56] J. Sommer-Larsen and A. Dolgov, ApJ 551, 608 (2001), arXiv:astro-ph/9912166.

[57] A. Kusenko, Phys. Rev. Lett. 97, 241301 (2006), hep-ph/0609081.

[58] J. Hidaka and G. M. Fuller, Phys. Rev. D 74, 125015 (2006), astro-ph/0609425.

[59] J. Hidaka and G. M. Fuller, Phys. Rev. D 76, 083516 (2007), arXiv:0706.3886.

[60] J. Stasielak, P. L. Biermann, and A. Kusenko, ApJ 654, 290 (2007), arXiv:astro-ph/0606435.

[61] M. Shaposhnikov (2007), 0708.3550.

[62] A. D. Dolgov and S. H. Hansen, Astropart. Phys. 16, 339 (2002), hep-ph/0009083.

[63] K. Abazajian, G. M. Fuller, and M. Patel, Phys. Rev. D 64, 023501 (2001), astro-ph/0101524.

[64] T. Asaka, M. Laine, and M. Shaposhnikov, JHEP 06, 053 (2006), hep-ph/0605209.

[65] T. Asaka, M. Laine, and M. Shaposhnikov, JHEP 01, 091 (2007), hep-ph/0612182.

[66] X.-d. Shi and G. M. Fuller, Phys. Rev. Lett. 82, 2832 (1999), astro-ph/9810076.

[67] M. Shaposhnikov, JHEP 08, 008 (2008), 0804.4542.

[68] M. Laine and M. Shaposhnikov, JCAP 6, 31 (2008), arXiv:0804.4543.

[69] M. Shaposhnikov and I. Tkachev, Phys. Lett. B639, 414 (2006), hep-ph/0604236.

[70] K. Petraki and A. Kusenko, Phys. Rev. D77, 065014 (2008), 0711.4646.

[71] K. Petraki, Phys. Rev. D 77, 105004 (2008), arXiv:0801.3470.

[72] M. Viel, J. Lesgourgues, M. G. Haehnelt, S. Matarrese, and A. Riotto, Phys. Rev. Lett. 97, 071301 (2006), astro$\mathrm{ph} / 0605706$.

[73] U. Seljak, A. Makarov, P. McDonald, and H. Trac, Phys. Rev. Lett. 97, 191303 (2006), astro-ph/0602430.

[74] M. Viel, G. D. Becker, J. S. Bolton, M. G. Haehnelt, M. Rauch, and W. L. W. Sargent, Phys. Rev. Lett. 100, 041304 (2008), arXiv:0709.0131.

[75] J. Dunkley et al. (WMAP) (2008), 0803.0586.

[76] A. Palazzo, D. Cumberbatch, A. Slosar, and J. Silk (2007), arXiv:0707.1495 [astro-ph].

[77] A. Boyarsky, J. Lesgourgues, O. Ruchayskiy, and M. Viel (2008), 0812.3256. 
[78] S. H. Hansen, J. Lesgourgues, S. Pastor, and J. Silk, MNRAS 333, 544 (2002), astro-ph/0106108.

[79] J. D. Simon and M. Geha, ApJ 670, 313 (2007), arXiv:0706.0516.

[80] G. Gilmore, M. I. Wilkinson, R. F. G. Wyse, J. T. Kleyna, A. Koch, N. W. Evans, and E. K. Grebel, ApJ 663, 948 (2007), arXiv:astro-ph/0703308.

[81] N. Bilic and R. D. Viollier, Phys. Lett. B 408, 75 (1997), arXiv:astro-ph/9607077.

[82] R. D. Viollier, Progress in Particle and Nuclear Physics 32, 51 (1994).

[83] N. F. Martin, J. T. A. de Jong, and H.-W. Rix, ArXiv e-prints 805 (2008), 0805.2945.

[84] M. Kuhlen, J. Diemand, and P. Madau, ApJ 671, 1135 (2007), 0705.2037.

[85] R. Cowsik and P. Ghosh, ApJ 317, 26 (1987).

[86] S. Tremaine, M. Henon, and D. Lynden-Bell, MNRAS 219, 285 (1986).

[87] J. Madsen, Phys. Rev. D 64, 027301 (2001), arXiv:astro-ph/0006074.

[88] D. Boyanovsky, H. J. de Vega, and N. G. Sanchez, Phys. Rev. D 77, 043518 (2008), arXiv:0710.5180.

[89] J. Binney and S. Tremaine, Galactic Dynamics: Second Edition (Galactic Dynamics: Second Edition, by James Binney and Scott Tremaine. ISBN 978-0-691-13026-2 (HB). Published by Princeton University Press, Princeton, NJ USA, 2008., 2008).

[90] S. H. Hansen, D. Egli, L. Hollenstein, and C. Salzmann, New Astronomy 10, 379 (2005), arXiv:astro-ph/0407111.

[91] M. Bolz, A. Brandenburg, and W. Buchmuller, Nucl.Phys. B 606, 518 (2001), hep-ph/0012052.

[92] V. S. Rychkov and A. Strumia, Phys. Rev. D75, 075011 (2007), hep-ph/0701104.

[93] S. Borgani, A. Masiero, and M. Yamaguchi, Phys. Lett. B386, 189 (1996), hep-ph/9605222.

[94] D. Lynden-Bell and R. Wood, MNRAS 138, 495 (1968).

[95] V. A. Antonov, Solution of the problem of stability of stellar system Emden's density law and the spherical distribution of velocities (Vestnik Leningradskogo Universiteta, Leningrad: University, 1962, 1962).

[96] C. Pryor and J. Kormendy, AJ 100, 127 (1990).

[97] S. Cole and C. Lacey, MNRAS 281, 716 (1996), arXiv:astro-ph/9510147.

[98] R. G. Carlberg, H. K. C. Yee, E. Ellingson, S. L. Morris, R. Abraham, P. Gravel, C. J. Pritchet, T. Smecker-Hane, F. D. A. Hartwick, J. E. Hesser, et al., ApJ 485, L13+ (1997), arXiv:astro-ph/9703107.

[99] S. H. Hansen and B. Moore, New Astronomy 11, 333 (2006).

[100] A. Zait, Y. Hoffman, and I. Shlosman, ApJ 682, 835 (2008), 0711.3791.

[101] E. Van Hese, M. Baes, and H. Dejonghe, ArXiv e-prints (2008), 0809.0901.

[102] J. H. An and N. W. Evans, ApJ 642, 752 (2006), arXiv:astro-ph/0511686.

[103] N. W. Evans, J. An, and M. G. Walker, ArXiv e-prints (2008), 0811.1488.

[104] L. E. Strigari, J. S. Bullock, M. Kaplinghat, J. Diemand, M. Kuhlen, and P. Madau, 704 (2007), 0704.1817.

[105] X. Wu (2007), astro-ph/0702233.

[106] L. E. Strigari, S. M. Koushiappas, J. S. Bullock, M. Kaplinghat, J. D. Simon, M. Geha, and B. Willman, ApJ 678, 614 (2008), arXiv:0709.1510.

[107] V. Belokurov, D. B. Zucker, N. W. Evans, J. T. Kleyna, S. Koposov, S. T. Hodgkin, M. J. Irwin, G. Gilmore, M. I. Wilkinson, M. Fellhauer, et al., ApJ 654, 897 (2007), arXiv:astro-ph/0608448.

[108] S. Koposov, V. Belokurov, N. W. Evans, P. C. Hewett, M. J. Irwin, G. Gilmore, D. B. Zucker, H. . Rix, M. Fellhauer, E. F. Bell, et al., ApJ 663, 948 (2007), 0706.2687.

[109] M. J. Irwin, V. Belokurov, N. W. Evans, E. V. Ryan-Weber, J. T. A. de Jong, S. Koposov, D. B. Zucker, S. T. Hodgkin, G. Gilmore, P. Prema, et al., ApJ 656, L13 (2007), arXiv:astro-ph/0701154.

[110] D. B. Zucker, V. Belokurov, N. W. Evans, J. T. Kleyna, M. J. Irwin, M. I. Wilkinson, M. Fellhauer, D. M. Bramich, G. Gilmore, H. J. Newberg, et al., ApJ 650, L41 (2006), arXiv:astro-ph/0606633.

[111] V. Belokurov, D. B. Zucker, N. W. Evans, M. I. Wilkinson, M. J. Irwin, S. Hodgkin, D. M. Bramich, J. M. Irwin, G. Gilmore, B. Willman, et al., ApJ 647, L111 (2006), arXiv:astro-ph/0604355.

[112] M. Mateo, E. Olszewski, D. L. Welch, P. Fischer, and W. Kunkel, AJ 102, 914 (1991).

[113] M. L. Mateo, ARA\&A 36, 435 (1998), astro-ph/9810070.

[114] A. Z. Bonanos, K. Z. Stanek, A. H. Szentgyorgyi, D. D. Sasselov, and G. Á. Bakos, AJ 127, 861 (2004), arXiv:astro$\mathrm{ph} / 0310477$.

[115] M. Dall'Ora, G. Clementini, K. Kinemuchi, V. Ripepi, M. Marconi, L. Di Fabrizio, C. Greco, C. T. Rodgers, C. Kuehn, and H. A. Smith, ApJ 653, L109 (2006), arXiv:astro-ph/0611285.

[116] M. G. Coleman, J. T. A. de Jong, N. F. Martin, H.-W. Rix, D. J. Sand, E. F. Bell, R. W. Pogge, D. J. Thompson, H. Hippelein, E. Giallongo, et al., ApJ 668, L43 (2007).

[117] J. T. A. de Jong, J. Harris, M. G. Coleman, N. F. Martin, E. F. Bell, H.-W. Rix, J. M. Hill, E. D. Skillman, D. J. Sand, E. W. Olszewski, et al., ApJ 680, 1112 (2008), arXiv:0801.4027.

[118] S. Okamoto, N. Arimoto, Y. Yamada, and M. Onodera, ArXiv e-prints 804 (2008), 0804.2976.

[119] M. G. Lee, H. S. Park, J.-H. Park, Y.-J. Sohn, S. J. Oh, I.-S. Yuk, S.-C. Rey, S.-G. Lee, Y.-W. Lee, H.-I. Kim, et al., AJ 126, 2840 (2003).

[120] L. Rizzi, E. V. Held, I. Saviane, R. B. Tully, and M. Gullieuszik, MNRAS 380, 1255 (2007), arXiv:0707.0521.

[121] M. Bellazzini, N. Gennari, F. R. Ferraro, and A. Sollima, MNRAS 354, 708 (2004), arXiv:astro-ph/0407444.

[122] M. Bellazzini, N. Gennari, and F. R. Ferraro, MNRAS 360, 185 (2005), arXiv:astro-ph/0503418.

[123] G. Pietrzyński, W. Gieren, O. Szewczyk, A. Walker, L. Rizzi, F. Bresolin, R.-P. Kudritzki, K. Nalewajko, J. Storm, M. Dall'Ora, et al., AJ 135, 1993 (2008), arXiv:0804.0347. 
[124] R. R. Muñoz, J. L. Carlin, P. M. Frinchaboy, D. L. Nidever, S. R. Majewski, and R. J. Patterson, ApJ 650, L51 (2006), arXiv:astro-ph/0606271.

[125] D. N. Spergel, R. Bean, O. Doré, M. R. Nolta, C. L. Bennett, J. Dunkley, G. Hinshaw, N. Jarosik, E. Komatsu, L. Page, et al., ApJS 170, 377 (2007), arXiv:astro-ph/0603449.

[126] A. Boyarsky, A. Neronov, O. Ruchayskiy, and M. Shaposhnikov, MNRAS 370, 213 (2006), astro-ph/0512509.

[127] A. Boyarsky, A. Neronov, O. Ruchayskiy, and M. Shaposhnikov, Phys. Rev. D 74, 103506 (2006), astro-ph/0603368.

[128] A. Boyarsky, A. Neronov, O. Ruchayskiy, M. Shaposhnikov, and I. Tkachev, Phys. Rev. Lett. 97, 261302 (2006), astro$\mathrm{ph} / 0603660$.

[129] S. Riemer-Sørensen, S. H. Hansen, and K. Pedersen, ApJ 644, L33 (2006), astro-ph/0603661.

[130] C. R. Watson, J. F. Beacom, H. Yuksel, and T. P. Walker, Phys. Rev. D74, 033009 (2006), astro-ph/0605424.

[131] A. Boyarsky, O. Ruchayskiy, and M. Markevitch, ApJ 673, 752 (2008), astro-ph/0611168.

[132] K. N. Abazajian, M. Markevitch, S. M. Koushiappas, and R. C. Hickox, Phys. Rev. D 75, 063511 (2007), arXiv:astro$\mathrm{ph} / 0611144$.

[133] A. Boyarsky, J. Nevalainen, and O. Ruchayskiy, A\&A 471, 51 (2007), astro-ph/0610961.

[134] A. Boyarsky, J. W. den Herder, A. Neronov, and O. Ruchayskiy, Astropart. Phys. 28, 303 (2007), astro-ph/0612219.

[135] A. Boyarsky, D. Iakubovskyi, O. Ruchayskiy, and V. Savchenko, MNRAS 387, 1361 (2008), arXiv:0709.2301.

[136] A. Boyarsky, D. Malyshev, A. Neronov, and O. Ruchayskiy, MNRAS 387, 1345 (2008), 0710.4922.

[137] G. R. Blumenthal, S. M. Faber, R. Flores, and J. R. Primack, ApJ 301, 27 (1986).

[138] O. Y. Gnedin, A. V. Kravtsov, A. A. Klypin, and D. Nagai, ApJ 616, 16 (2004), arXiv:astro-ph/0406247.

[139] D. N. C. Lin and S. M. Faber, ApJ 266, L21 (1983).

[140] B. Moore, Nature 370, 629 (1994).

[141] C. Mastropietro, B. Moore, L. Mayer, V. P. Debattista, R. Piffaretti, and J. Stadel, MNRAS 364, 607 (2005), arXiv:astro$\mathrm{ph} / 0411648$.

[142] L. Mayer, C. Mastropietro, J. Wadsley, J. Stadel, and B. Moore, MNRAS 369, 1021 (2006), arXiv:astro-ph/0504277.

[143] L. Mayer, S. Kazantzidis, C. Mastropietro, and J. Wadsley, Nature 445, 738 (2007), arXiv:astro-ph/0702495.

[144] J. I. Read, A. P. Pontzen, and M. Viel, MNRAS 371, 885 (2006), arXiv:astro-ph/0606391.

[145] T. Naab, P. H. Johansson, J. P. Ostriker, and G. Efstathiou, ApJ 658, 710 (2007), arXiv:astro-ph/0512235.

[146] E. Bertschinger (1995), astro-ph/9506070.

[147] A. Klypin and J. Holtzman (1997), astro-ph/9712217.

[148] A. Klypin (2000), astro-ph/0005502.

[149] J. E. Taylor and J. F. Navarro, Astrophys. J. 563, 483 (2001), astro-ph/0104002.

[150] S. Peirani, F. Durier, and J. A. de Freitas Pacheco, MNRAS 367, 1011 (2006), arXiv:astro-ph/0512482.

[151] S. Peirani and J. A. de Freitas Pacheco, ArXiv Astrophysics e-prints (2007), astro-ph/0701292.

[152] E. Romano-Díaz, Y. Hoffman, C. Heller, A. Faltenbacher, D. Jones, and I. Shlosman, ApJ 657, 56 (2007).

[153] Y. Hoffman, E. Romano-Díaz, I. Shlosman, and C. Heller, ApJ 671, 1108 (2007), arXiv:0706.0006.

[154] E. Romano-Diaz, A. Faltenbacher, D. Jones, C. Heller, Y. Hoffman, and I. Shlosman, ApJ 637, L93 (2006), arXiv:astro$\mathrm{ph} / 0508272$.

[155] D. Gorbunov, A. Khmelnitsky, and V. Rubakov (2008), 0805.2836.

[156] D. Boyanovsky, Phys. Rev. D78, 103505 (2008), 0807.0646.

[157] D. Gorbunov, A. Khmelnitsky, and V. Rubakov, JCAP 0810, 041 (2008), 0808.3910.

[158] L. D. Landau and E. M. Lifshitz, The classical theory of fields (Course of theoretical physics - Pergamon International Library of Science, Technology, Engineering and Social Studies, Oxford: Pergamon Press, 1975, 4th rev.engl.ed., 1975).

[159] L. D. Landau and E. M. Lifshitz, Statistical physics. Pt.1, Pt.2 (Course of theoretical physics, Pergamon International Library of Science, Technology, Engineering and Social Studies, Oxford: Pergamon Press, 1980 - c1980, 3rd rev.and enlarg. ed., 1980). 